\title{
Spin-bias driven magnetization reversal and nondestructive detection in a single molecular magnet
}

\author{
Hai-Zhou Lu (卢海舟), ${ }^{1}$ Bin Zhou (周斌), ${ }^{1,2}$ and Shun-Qing Shen (沈顺清) ${ }^{1}$ \\ ${ }^{1}$ Department of Physics and Centre of Theoretical and Computational Physics, The University of Hong Kong, Pokfulam Road, \\ Hong Kong, China \\ ${ }^{2}$ Department of Physics, Hubei University, Wuhan 430062, China
}

(Received 15 December 2008; published 13 May 2009)

\begin{abstract}
The magnetization reversal in a single molecular magnet (SMM) weakly coupled to an electrode with spin-dependent splitting of chemical potentials (spin bias) is theoretically investigated by means of the rate equation. A microscopic mechanism for the reversal is demonstrated by the avalanche dynamics at the reversal point. The magnetization as a function of the spin bias shows hysteresis loops tunable by the gate voltage and varying with temperature. The nondestructive measurement to the orientation of giant spin in SMM is presented by measuring the fully polarized electric current in the response to a small spin bias. For $\mathrm{Mn}_{12} \mathrm{ac}$ molecule, its small transverse anisotropy only slightly violates the results above. The situation when there is an angle between the easy axis of the SMM and the spin-quantization direction of the electrode is also studied.
\end{abstract}

DOI: 10.1103/PhysRevB.79.174419

PACS number(s): 75.50.Xx, 75.60.Jk, 72.25.Hg

\section{INTRODUCTION}

Magnetization reversal driven by spin-polarized electric current had attracted considerable interests over the last decade..$^{1-5}$ Recent experiments ${ }^{6,7}$ demonstrated that a pure spin current accompanied by no net charge current can also be used to reverse the magnetization of a ferromagnetic particle. In this setup, the particle is attached to a nonmagnetic metal wire, in which the chemical potentials of two spin components are split by using the nonlocal spin injection technique..$^{8-11}$ By changing only the direction of injection current, the sign of the splitting can be reversed, leading to the magnetization reversal in both orientations. ${ }^{7}$ Application of only pure spin current is appealing for spintronics devices because it helps to reduce critical currents, Joule heat, noise, etc.

Meanwhile, another family of intensively studied nanoscale magnetic materials, the single molecular magnets (SMM), ${ }^{12-19}$ was reported recently to be trapped in a typical field effect transistor geometry, allowing electronic transport measurement to be performed on an individual SMM with great tunability. ${ }^{20-23}$ The experiment progresses inspired many transport theories of SMMs, including magnetic signatures of SMMs in transport, ${ }^{24-27}$ Kondo effect, ${ }^{28-32}$ Berry phase, ${ }^{29,33}$ full counting statistics, ${ }^{34}$ quantum computing, ${ }^{35}$ cotunneling, ${ }^{36}$ and vibrational excitation. ${ }^{31}$

Ion spins of magnetic metal in an SMM are interlocked to form a collective giant spin, whose two maximally magnetized ground states orient to opposite directions due to uniaxial anisotropy, a property that implies to be a promising candidate for high-density information storage. Therefore, one of the important issues is how to manipulate and measure the magnetization of an SMM, ${ }^{25,37-42}$ i.e., the processes of writing and reading qubit encoded by $\mathrm{SMM},{ }^{43,44}$ using transport approaches. It has been discussed that spin accumulation can be induced by charge current. ${ }^{26}$ Besides, it has been proposed that spin-polarized electric current injected from ferromagnetic electrodes can be used to switch the magnetization of SMM. . $^{37,39,41,42}$
Gold electrode is used in all the SMM transport experiments by far, ${ }^{20-23}$ which is also among the metals $(\mathrm{Au}, \mathrm{Ag}$, $\mathrm{Al}, \mathrm{Cu}$ ) employed in nonlocal spin injection technique (for a review, see Ref. 45 and references therein). Therefore, it is natural to expect that the magnetization reversal achieved in ferromagnetic particle ${ }^{6,7}$ could also be realized in SMM using the nonlocal spin injection. After all, the magnetic moment of SMM is much smaller than that of ferromagnetic particle. Although generating considerable spin-dependent splitting of chemical potentials with high efficiency remains a challenge, it has been demonstrated experimentally by many other approaches such as the spin Hall effect, ${ }^{46,47}$ the spin pumping effect, ${ }^{48,49}$ and incidence of polarized light into two-dimensional electron gas. ${ }^{50-54}$

Motivated by the progresses in both the nonlocal spin injection and SMMs, in this work, we demonstrate theoretically that a pure spin current induced by the spin-dependent splitting of chemical potential (spin bias) in a nonmagnetic electrode $^{55-59}$ is enough to reverse the SMM magnetization, as shown in Fig. 1, where $V$ phenomenologically denotes the spin dependent splitting of the Fermi levels for $\uparrow$ and $\downarrow$ elec-

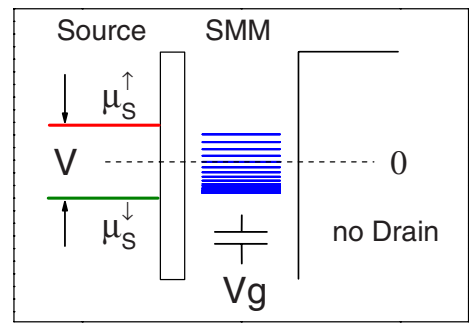

FIG. 1. (Color online) Schematic of energy configurations of our setup to manipulate the magnetization of an SMM. The splitting between $\uparrow$ and $\downarrow$ Fermi levels of the only source lead is phenomenologically denoted as $V$. Throughout the work the middle point of $\mu_{S}^{\uparrow / \downarrow}$ is set at 0 so that $\mu_{S}^{\uparrow / \downarrow}= \pm \frac{V}{2}$. The horizontal lines in the SMM region correspond to resonant energies to add an extra electron into the SMM via transitions from state $|0, m\rangle$ to $\left|1, m \pm \frac{1}{2}\right\rangle^{-}$, which is tunable with respect to $\mu_{S}^{\uparrow / \downarrow}$ by the gate voltage $V_{g}$. 
trons in the electrode, i.e., $\mu_{S}^{\uparrow / \downarrow}= \pm V / 2$. We find that in the context of the spin bias: (i) to reverse the SMM magnetization, only one nonmagnetic electrode is needed. Neither magnetic field ${ }^{37}$ nor magnetic contact ${ }^{37,39,41}$ is required. (ii) Only a pure spin current without accompanying a net electric current flows in the process of reversal (Fig. 5), which avoids the relaxation of magnetization induced by electric current. ${ }^{37}$ (iii) It sheds a light on the mechanism of magnetization reversal from a microscopic point of view and may be extended to mesoscopic magnetic particles or films. ${ }^{6,7}$

Moreover, we will discuss, in the context of using spin bias, several effects not addressed or not clarified in the previous literatures on the current-induced magnetic reversal in SMM. (i) By analyzing the transition energy spectrum (Fig. 3 ), we find that the activation energy at which the magnetic reversal starts is determined not only by the highest, ${ }^{37,41}$ but also by the lowest transition energy and is tunable by the gate voltage. (ii) The SMM magnetization show magnetic hysteresis loop when scanning the spin bias back and forth. The hysteresis loop can be tuned by the gate voltage and shrinks with increasing temperature (Fig. 4). (iii) The avalanche dynamics at the magnetic reversal point is demonstrated (Fig. 5), which supports a microscopic magnetization reversal mechanism. (iv) We show that the ground-state orientation of the giant spin in SMM can be read out noninvasively by measuring the charge current through SMM driven by a small spin bias (Fig. 7). (v) The effect of weak transverse anisotropy is considered. (vi) The situation when there is an angle between the SMM easy axis and the spinquantization direction of the electrode is discussed.

The paper is organized as follows. First, we will show that the reversal mechanism is irrelevant to specific model used in Sec. II. The model and general formalism of theoretical approach will be introduced in details in Sec. III. In Sec. IV, we present the numerical simulations of the hysteresis loops tunable by the gate voltage, the avalanche dynamics at the magnetic reversal point, and the nondestructive detection to the orientation of the giant spin. In Sec. V, the correction by the transverse anisotropy is considered. In Sec. VI, the case when SMM easy axis is not collinear with the spinquantization direction of the electrode is investigated. Finally, a summary is presented to compare the advantages of the present work to the existing proposals.

\section{MODEL-IRRELEVANT MAGNETIZATION REVERSAL MECHANISM}

By far, many models are proposed to describe SMM with extra electrons added into it. These models include the giant spin model, ${ }^{20,21,25,37,38,41}$ multi-ion model, ${ }^{60}$ and those based on the density-functional theory. ${ }^{61,62}$

Despite the model employed, one can always select out two branches of many-body states of SMM. One is for the neutral SMM, the other is for when the SMM is charged with an extra electron. Assume the total angular momentum of the ground state of the neutral SMM is $S$, which has $2 S+1$ states for different $z$ component of total angular momentum, denoted as $|0, m\rangle(m \in[-S, S])$. Adding the extra electron is like coupling two angular momenta, leading to two possible

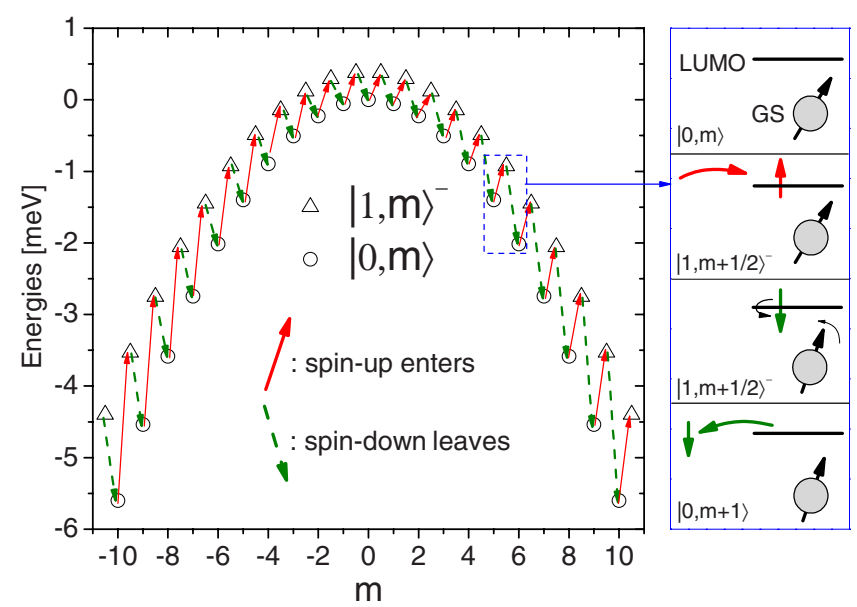

FIG. 2. (Color online) Left: The two branches of molecular many-body states considered in the simulations of this work. The basic parameters are given in Sec. III B. $V_{g}=-20 \mathrm{mV}$. Arrows indicate all the steps required to reverse the SMM magnetization from $-21 / 2$ to 21/2. Right (four panels): The schematic of a single step that increases the SMM magnetization by 1 . This mechanism is supported by the simulation shown in Figs. 5 and 6.

ground-state total angular momenta for the charged branches, $S \pm \frac{1}{2}$, respectively. For simplicity, we assume the total angular momentum of the ground state of the charged branch is $S+\frac{1}{2}$, which has $2 S+2$ states, denoted as $|1, m\rangle\left(m \in\left[-S-\frac{1}{2}, S+\frac{1}{2}\right]\right)$. Because of the easy-axis anisotropy, the ground states of the two branches are $|0, \pm S\rangle$ and $\left|1, \pm\left(S+\frac{1}{2}\right)\right\rangle$, respectively. As an example, two such branches with uniaxial anisotropy barriers are shown in Fig. 2 using the giant spin model proposed by Timm and Elste. ${ }^{37,38}$

Suppose one wants to reverse the giant spin originally orienting antiparallel with the $z$ axis, i.e., at the state $|1,-21 / 2\rangle$ or $|0,-10\rangle$, to parallel orientation, i.e., the state $|1,21 / 2\rangle$ or $|0,10\rangle$. By connecting SMM to the lead, one can generate a sequence of transitions that charge SMM with spin-up electrons and discharge SMM with spin-down electrons

$$
\begin{aligned}
|1,-21 / 2\rangle & \rightarrow|0,-10\rangle \rightarrow|1,-19 / 2\rangle \rightarrow \ldots \rightarrow|1,-11 / 2\rangle \\
& \rightarrow \ldots \rightarrow|0,0\rangle \rightarrow \ldots \rightarrow|1,11 / 2\rangle \rightarrow \ldots \\
& \rightarrow|1,19 / 2\rangle \rightarrow|0,10\rangle \rightarrow|1,21 / 2\rangle,
\end{aligned}
$$

as shown in Fig. 2. During each of these transitions, the magnetic moment of an electron spin is transferred to SMM by either adding an spin- $\uparrow$ electron into or removing a spin- $\downarrow$ electron from SMM. The right four panels in the Fig. 2 depict a typical step in which an $\uparrow$ spin enters, flips to $\downarrow$ spin owing to the exchange coupling while increases the giant spin orientation by one unit, and escapes from SMM. Such a step repeats until the giant spin orientation is reversed.

Energetically, to generate this charging-discharging sequence, the Fermi level for the spin-up electrons in the lead should be higher than all the transition energies of adding an spin-up electron from a neutral state $|0, m\rangle$ to a charged state $\left|1, m+\frac{1}{2}\right\rangle$, i.e., 


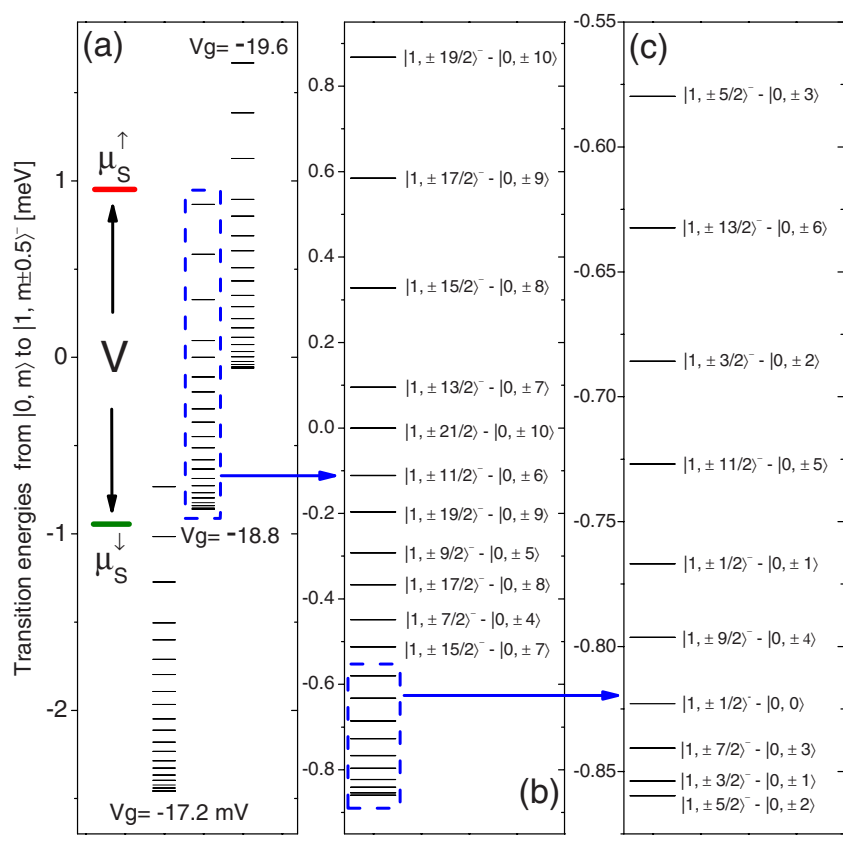

FIG. 3. (Color online) (a) Resonant energies to add an extra electron into SMM via transitions from state $|0, m\rangle$ to $\left|1, m \pm \frac{1}{2}\right\rangle^{-}$for three different $V_{g}$. The middle point of $\mu_{S}^{\uparrow / \downarrow}$ is set as energy zero point so that $\mu_{S}^{\uparrow / \downarrow}= \pm V / 2$. (b) Zoom-in when $V_{g}=-18.8 \mathrm{mV}$, where the middle point of the entire spectrum is aligned with 0 , i.e., the middle point of the Fermi levels $\mu_{S}^{\uparrow / \downarrow}$. (c) Zoom-in of the lowest transitions in the middle panel. The notation $\left|1, m \pm \frac{1}{2}\right\rangle^{-}-|0, m\rangle$ is short for $E_{|1, m \pm 1 / 2\rangle^{-}}-E_{|0, m\rangle}$. Note that $E_{|1,-(m \pm 1 / 2)\rangle^{-}}-E_{|0,-m\rangle}=E_{|1, m \pm 1 / 2\rangle^{-}}-E_{|0, m\rangle}$ are degenerate in the absence of magnetic field. Basic parameters are given in Sec. III B.

$$
\mu_{S}^{\uparrow}>E_{|1, m+1 / 2\rangle}-E_{|0, m\rangle},
$$

while the spin-down Fermi level of the lead should be lower than all the transition energies of adding an spin-down electron from a neutral state $|0, m\rangle$ to a charged state $\left|1, m-\frac{1}{2}\right\rangle$, i.e.,

$$
\mu_{S}^{\downarrow}<E_{|1, m-1 / 2\rangle}-E_{|0, m\rangle} .
$$

Because of the anisotropy of SMM, the spectrum of the transitions for all the possible $m$ has a finite width, as shown in Fig. 3. Therefore, the splitting of $\mu_{\uparrow}$ and $\mu_{\downarrow}$, i.e., the spin bias, must be large enough to overcome this spectrum width. This thereby defines a threshold spin bias for the reversal.

In the process of the reversal, only $\uparrow$ electrons enter SMM while only $\downarrow$ electrons leave SMM at almost the identical rate. As a result, a nearly pure spin current, instead of electric current, flows between the lead and the SMM. Once the reversal is accomplished, i.e., $|1,21 / 2\rangle$ is occupied, no more $\uparrow(\downarrow)$ electron can enter (leave) and the pure spin current decays to zero. The magnetization reversal mechanism discussed above is supported by the simulation results shown in Fig. 5.

Note that the neutral and charged branches employed in the above reversal mechanism universally exist not only within the giant spin approximation, ${ }^{20,21,25,37,38,41}$ but also in the multi-ion model ${ }^{60}$ and density-functional theory. ${ }^{61,62}$ In the multi-ion model of Lehmann and Loss, ${ }^{60}$ the case of ferromagnetic inter-ion interaction corresponds to the type of SMM discussed in the present work. The green dash-dotted lines in Fig. 1(a) of their paper describe the transitions between the neutral and charged branches with the difference of the total angular momentum by $1 / 2$. Recent densityfunction theory calculation also concludes that the total angular momenta for the ground-state neutral and anionic branches are 10 and 21/2, respectively. ${ }^{62}$ Therefore the above reversal mechanism should be universally described by most models proposed by far. Later we will also show that the above mechanism is not qualitatively affected by the transverse anisotropy (Sec. V) and noncollinearity (Sec. VI).

\section{GENERAL FORMALISM}

\section{A. Model}

In this work, we want to focus on the possibility of using spin bias and what we need are one neutral branch and one charged branch as discussed above. Specifically, we adopt the model proposed by Timm and Elste ${ }^{37,38}$ to describe SMM, which consists of the lowest unoccupied molecular orbital (LUMO) and the phenomenological giant spin (GS) $\mathbf{S}$ of the molecule

$$
\begin{aligned}
H_{\mathrm{SMM}}= & \left(\epsilon_{0}-e V_{g}\right) \sum_{\sigma \in\{\uparrow, \downarrow\}} n_{\sigma}+U n_{\uparrow} n_{\downarrow}-\left(D+\delta D \sum_{\sigma} n_{\sigma}\right) S_{z}^{2} \\
& +H^{\prime}+E_{\mathrm{ion}}\left(V_{g}\right)-J \mathbf{S} \cdot \mathbf{S}
\end{aligned}
$$

where the first two terms depict the LUMO, $n_{\sigma}=d_{\sigma}^{\dagger} d_{\sigma}$, and $d_{\sigma}\left(d_{\sigma}^{\dagger}\right)$ are the annihilation (creation) operators for the LUMO, whose on-site energy is tunable by a gate voltage $V_{g}$. $-e$ is the electron charge. $U$ is the on-site Coulomb repulsion.

The third and forth terms are for the anisotropy of GS, where $D$ describes the easy-axis anisotropy and $\delta D$ accounts for the correction to the easy-axis anisotropy by the occupation of LUMO. $H^{\prime}$ describes the transverse anisotropy. We formally consider its possible leading terms

$$
H^{\prime}=B_{2}\left(S_{+}^{2}+S_{-}^{2}\right)+B_{4}\left(S_{+}^{4}+S_{-}^{4}\right) .
$$

For $\mathrm{Mn}_{12}$ ac, there is usually only $B_{4}$ term. We also include $B_{2}$ term because we want to investigate the effect of the transverse terms in a general way. For $\mathrm{Mn}_{12}$ ac, $B_{4}$ are several orders smaller than the easy-axis anisotropy $D$ (Table 4.1 of Ref. 19). Therefore, we assume that extra electron brings no correction to them. Following most experiments and theories by far, ${ }^{20,21,25,37,38,60}$ we assume the extra electrons change only the magnitude of the easy-axis anisotropy.

The fifth term is the energy of ions that form the giant spin, which is also proportional to $V_{g}$ in the same way as LUMO. The last term describes the Hund's rule coupling $J$ between the giant spin $\mathbf{S}$ and the electron spin $\mathbf{S}$ in the LUMO $\mathbf{s}=\frac{1}{2} \sum d_{\alpha}^{\dagger}(\vec{\sigma})_{\alpha \beta} d_{\beta}$, where $\vec{\sigma}$ are the vector of Pauli matrices.

The Hamiltonian for the electrodes used to probe SMM reads 


$$
H_{\text {lead }}=\sum_{k, \alpha, \tau} \epsilon_{k \alpha} c_{k \alpha \tau}^{\dagger} c_{k \alpha \tau},
$$

where $c_{k \alpha \tau}^{\dagger}\left(c_{k \alpha \tau}\right)$ is the creation (annihilation) operator for a continuous state in the $\alpha(\in\{\mathrm{S}, \mathrm{D}\})$ lead with energy $\epsilon_{k \alpha}$ and spin $\tau \in\{+,-\}$. In reality, there should be an angle $\theta$ between the easy axis of SMM and the spin orientation in the electrodes, so the spin quantization in SMM is denoted $\sigma \in\{\uparrow, \downarrow\}$ and in the lead as $\tau \in\{+,-\}$. The operator for spin- $\tau$ electron in the lead is related to spin- $\sigma$ operator through an $\mathrm{SU}(2)$ rotation.

The tunneling between the LUMO and the electrodes is described by

$$
\begin{aligned}
H_{T}= & \sum_{k, \alpha} V_{k \alpha}\left[\left(\cos \frac{\theta}{2} c_{k \alpha+}^{\dagger}-\sin \frac{\theta}{2} c_{k \alpha-}^{\dagger}\right) d_{\uparrow}\right. \\
& \left.+\left(\sin \frac{\theta}{2} c_{k \alpha+}^{\dagger}+\cos \frac{\theta}{2} c_{k \alpha-}^{\dagger}\right) d_{\downarrow}\right]+ \text { H.c. },
\end{aligned}
$$

where $\theta \in[0, \pi / 2]$. We set the easy axis of the SMM as $z$ axis. For $\theta \in[\pi / 2, \pi]$ one just reverses the positive direction of $z$ axis. In short, the total Hamiltonian for the system we are studying is

$$
H_{\text {total }}=H_{\text {SMM }}+H_{\text {lead }}+H_{T} .
$$

We believe that the Hamiltonian employed in this work captures the physics required for the magnetization reversal, though it is a simplified model. The physical picture of this model can be understood as follows. In a $\mathrm{Mn}_{12}$ ac molecule, eight spin-2 $\mathrm{Mn}^{3+}$ ions and four spin- $-\frac{3}{2} \mathrm{Mn}^{4+}$ ions are exchange coupled. As a good quantum number, their total angular momentum may adopt many values, referred as different branches. The branch with the lowest energy consists of 21 states with a total angular momentum $S=10$. The branches with other values of total angular momentum are much higher in energy. Due to the anisotropy along the easy axis, two degenerate ground states of $S=10$ branch are the states with $z$ component of the total angular momentum $S_{z}= \pm S$, respectively. In this sense the system is regarded as a giant spin of $S=10$ and is simply described by the term $-D S_{z}^{2}$. By adding an extra electron, the energy of the molecule changes in several aspects: (i) The first is the on-site and charging energy to add this electron. This is described by $\epsilon_{0} \Sigma_{\sigma} n_{\sigma}$. If we set the Fermi level of the lead as the reference point, this energy can be compensated by the gate voltage, so it is absorbed into the term $\left(\epsilon_{0}-e V_{g}\right) \Sigma_{\sigma} n_{\sigma}$. The $U$ term is due to adding the second excess electron to the same LUMO. To simplify the problem, we assume the second electron will also occupy the same LUMO and exclude the possibility to occupy other states. This is purely theoretical simplification and is believed not to affect qualitatively the physical consequences in the present problem. We have to emphasize that the energy of the ions $E_{\text {ion }}\left(V_{g}\right)$ is also tunable to the gate voltage $V_{g}$ in the same way as the excess electron. It does not have to explicitly appear in the Hamiltonian because it is counted in the energy of each many-body state of the SMM, thus can be discarded. (ii) Second, the added electron will interact with the giant spin to form spin $S+1 / 2$ and $S-1 / 2$ branches of states. The energy difference between these two branches can be characterized by the term $-J \mathbf{S} \cdot \mathbf{s}$, where $J$ can be found by the splitting between the two branches by using first-principle calculation, ${ }^{61}$ because the splitting is around 2JS. (iii) The third is the correction of the anisotropy due to the excess electron. Because the angular momentum of electron spin is much smaller than that of the giant spin, the variation of anisotropy in the presence of the excess electron, which mainly leads to a curvature change in the energy spectrum, can be roughly absorbed into the correction parameter $\delta D$.

Either the giant spin model proposed by Timm and Elste $^{37,38}$ or by Romeike and co-workers ${ }^{20,25}$ describes the above physical picture. Both characterize the many-body eigenstates by the electron occupation, the total angular momentum (note that $\nu= \pm$ correspond to the total angular momentum $=S \mp \frac{1}{2}$ ), and the $z$ component of total angular momentum. When calculating the matrix elements $\left\langle i\left|d_{\sigma}\right| j\right\rangle$, the Clebsh-Gordan coefficients ${ }^{25}$ correspond to the linear combination coefficients ${ }^{37} \alpha_{m}^{ \pm}, \beta_{m}^{ \pm}$. Most importantly, both model are able to capture the main features of the experiments, e.g., the sophisticated magnetic excitations and the negative differential conductance observed in the experiments. ${ }^{20}$

Besides, Lehmann and Loss ${ }^{60}$ think that the inclusion of the excess electron with respect to the uncharged SMM should start with a multi-ion model, in which $N$ ion sites are considered, each with an ion spin $s$. Nearest ions are coupled by either ferromagnetic or antiferromagnetic exchange interaction. The excess electron can occupy and hop among any of these ion sites. The Hund's rule coupling between the excess electron and each ion is local, as well as the anisotropy. When considering antiferromagnetic inter-ion coupling, the ground state adopts a zero total angular momentum and apparently is not the case for $\mathrm{Mn}_{12}$ ac (but valid for other SMMs, such as $\mathrm{Mn}_{4}$ dimer $^{63,64}$ ). The spatial selection rules they predicted mainly occur for the antiferromagnetic case, thus will not be considered in this work. When considering ferromagnetic inter-ion coupling, the ground state adopts a maximal angular momentum Ns. They considered only one excess electron. In this case, the electron is free to hop and couple to all the ions. As a result, the local Hund's rule coupling and anisotropy give rise to global giant spin properties. In a word, the single excess electron and ferromagnetic inter-ion case of Lehmann and Loss's model shares the same spirit of those by Timm and Elste ${ }^{37,38}$ and Romeike and co-workers. ${ }^{20,25}$

The spatial selection rules are also predicted by using the density-functional theory. ${ }^{62}$ However, because of the lead $(\sim 100 \mathrm{~nm})$ used in the experiment ${ }^{20}$ is much wider than the size of the molecule $(<5 \mathrm{~nm})$, we think the spatial selection rules, which need precise contacts between the lead and the ion sites of the molecule, could be smeared in realistic samples.

Though the coexistence of electron-phonon interaction and magnetic excitation is observed, ${ }^{20,21}$ the phonon frequency is beyond the energy scale of the current work. For example, the phonon frequency observed by Heersche et $a .^{20}$ is about $14 \mathrm{meV}$, while the magnetic excitations observed $^{20,21}$ or in this work (Fig. 3) and the spin bias (Fig. 4) are of order of meV. Therefore, we do not consider electron-phonon interaction and its related effect in this 

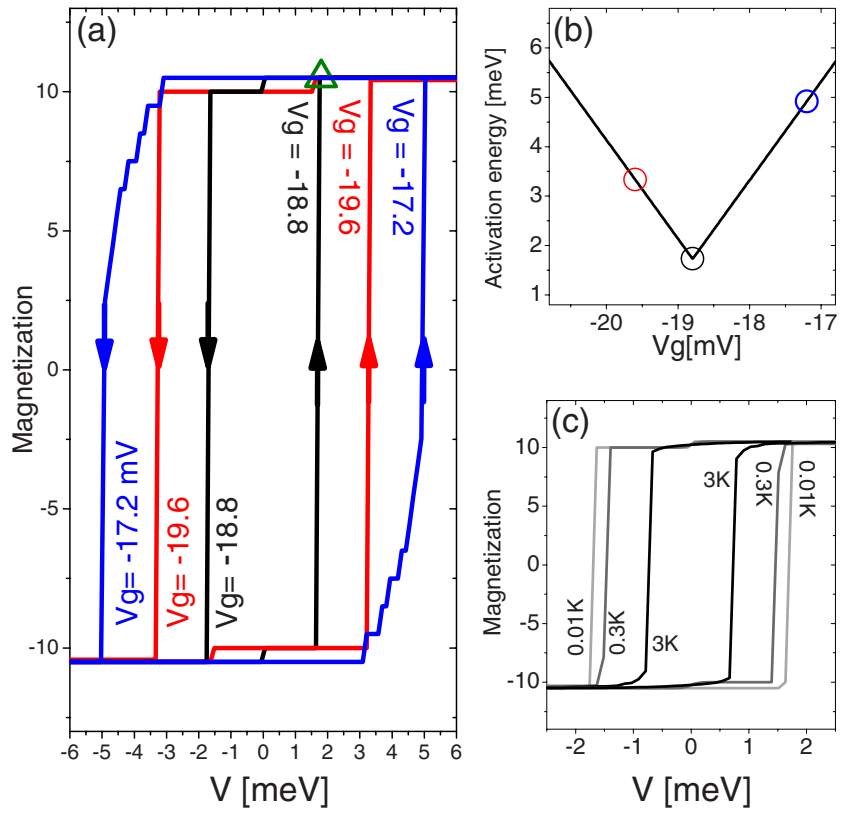

FIG. 4. (Color online) (a) Magnetic hysteresis loops for different gate voltage $V_{g}$, when scanning the spin bias $V$ back and forth. $T$ $=0.01 \mathrm{~K}$. Arrows indicate the scanning direction of $V$. The scanning is assumed to be slow enough to allow the system relax to steady state. The triangle corresponds to the magnetic reversal point for the simulation in Figs. 5 and 6. (b) The activation energy vs $V_{g}$. It consists of two slopes connecting at $V_{g}=-18.8 \mathrm{mV}$. The left slope is determined by the transition energy $E_{|1, \pm 19 / 2\rangle^{-}}-E_{|0, \pm 10\rangle}$; the right slope is determined by the transition energy $E_{|1, \pm 5 / 2\rangle^{-}}-E_{|0, \pm 2\rangle}$. Circles correspond to $V_{g}=-17.2,-18.8$, and $-19.6 \mathrm{mV}$ in the left panel. (c) Temperature-dependent magnetic hysteresis loops as a function of $V$ for $V_{g}=-18.8 \mathrm{mV}$. Results for experimental temperatures $3 \mathrm{~K}$ (Ref. 20) and $0.3 \mathrm{~K}$ (Ref. 21) are presented. Other parameters are given in Sec. III B.

work, e.g., Franck-Cordon blockade ${ }^{65}$ or thermal-activated effect induced by spin-phonon interaction. ${ }^{66}$

\section{B. SMM states in absence of transverse anisotropy and parameters for simulations}

We will use the eigenstates of $H_{\mathrm{SMM}}$ when $B_{2}=B_{4}=0$ as unperturbed states. ${ }^{37}$ The transverse anisotropy will be taken into account by perturbation for small $B_{2}$ and $B_{4}$.

When $B_{2}=B_{4}=0, H_{\mathrm{SMM}}$ leads to four branches of states for the isolated SMM denoted by $|n, m\rangle^{\nu}$, where $n(=0,1,2)$ is the LUMO occupation and $m$ is the quantum number for $\left(S_{z}+s_{z}\right)$, the $z$ component of total angular moment. ${ }^{37}$ Degeneracy index $\nu$ only appears when $n=1$. The four branches are:

The empty branch

$$
|0, m\rangle \equiv|0\rangle_{\mathrm{LUMO}} \otimes|m\rangle_{\mathrm{GS}}
$$

where $m \in[-S, S]$.

The two singly-occupied branches

$$
\begin{aligned}
|1, m\rangle^{ \pm} \equiv & \alpha_{m}^{ \pm}|\downarrow\rangle_{\mathrm{LUMO}} \otimes\left|m+\frac{1}{2}\right\rangle_{\mathrm{GS}}+\beta_{m}^{ \pm}|\uparrow\rangle_{\mathrm{LUMO}} \otimes \mid m \\
& \left.-\frac{1}{2}\right\rangle_{\mathrm{GS}},
\end{aligned}
$$

where $m \in\left[-S-\frac{1}{2}, S+\frac{1}{2}\right]$ for $\nu=-, m \in\left[-S+\frac{1}{2}, S-\frac{1}{2}\right]$ for $\nu=+$.

The doubly-occupied branch

$$
|2, m\rangle \equiv|\uparrow \downarrow\rangle_{\mathrm{LUMO}} \otimes|m\rangle_{\mathrm{GS}},
$$

where $m \in[-S, S]$.

One can refer to Fig. 2 of Ref. 41 to have a direct impression of these four branches. But different from Ref. 41, in this work the higher two branches are far above the lower two branches because of large $J$ and $U$.

We adopt the parameters based on recent experiments and first-principles calculations for $\operatorname{Mn}_{12} \mathrm{ac}(S=10)$ as $D=0.056 \mathrm{meV}, \delta D=-0.008 \mathrm{meV}^{20}$ and $J=3.92 \mathrm{meV} .{ }^{27,61}$ Because $\epsilon_{0}$ can be compensated by $V_{g}$, we set $\epsilon_{0}=0$ for convenience. We choose $U=25 \mathrm{meV}$, which is comparable to the width of Coulomb diamond in experiments. ${ }^{20,21}$ For the above parameters (large $J$ and $U$ ), the two highest branches $|2, m\rangle$ and $|1, m\rangle^{+}$are neglected in the present work because the branch $|1, m\rangle^{+}$is about $2 S J$ (about several tens of meV) above the branch $|1, m\rangle^{-}$and the branch $|2, m\rangle$ is even higher. In the following numerical simulations, we consider only the branches $|0, m\rangle$ and $|1, m\rangle^{-}$. By choosing suitable gate voltage $V_{g}$, these two branches can be nearly degenerate with respect to the Fermi levels of the leads. ${ }^{25}$ A typical situation of $V_{g}=-20 \mathrm{mV}$ is shown in Fig. 2.

\section{Perturbative correction to SMM states by transverse anisotropy}

For $\mathrm{Mn}_{12}$ ac, the transverse anisotropies $B_{2}$ and $B_{4}$ are several orders smaller than the easy-axis anisotropy $D$ (Table 4.1 of Ref. 19). For $B_{2} \ll D / S^{2}$ and $B_{4} \ll D / S^{4}$, they can be taken into account by the standard perturbation calculation. Note that degenerate states such as $|0, \pm 1\rangle$ and $|0, \pm 2\rangle$ are coupled by $H^{\prime}$, so one has to perform a degenerate perturbation calculation. We consider the first-order correction to the states and the second order to their energies (please refer to Appendix B for details).

In the presence of weak $B_{2}$ and $B_{4}$, the eigenstates can only be approximately labeled by the quantum number $m$ of $\left(S_{z}+s_{z}\right)$ and becomes a linear combination of all the states with the same LUMO occupation, ${ }^{25}$ i.e.,

$$
\begin{gathered}
|0, m\rangle_{p}=\sum_{m^{\prime}=-S}^{S} C_{m^{\prime}, m}^{0}\left|0, m^{\prime}\right\rangle, \\
|1, m\rangle_{p}^{-}=\sum_{m^{\prime}=-S-(1 / 2)}^{S+(1 / 2)} C_{m^{\prime}, m}^{-}\left|1, m^{\prime}\right\rangle^{-}+\sum_{m^{\prime}=-S+(1 / 2)}^{S-(1 / 2)} C_{m^{\prime}, m}^{+}\left|1, m^{\prime}\right\rangle^{+},
\end{gathered}
$$

where $p$ stands for perturbed states by $B_{2}$ and $B_{4}$. One can expect that $C_{m, m}^{0,-} \sim 1$, i.e., $|0, m\rangle_{p}$, are mainly contributed by $|0, m\rangle$ and $|1, m\rangle_{p}^{-}$by $|1, m\rangle^{-}$. 
The projection of magnetization along $z$ axis for the perturbed states are obtained, for the branch $|0, m\rangle_{p}$,

$$
m_{p}=\sum_{m^{\prime}=-S}^{S}\left|C_{m^{\prime}, m}^{0}\right|^{2} m^{\prime},
$$

and for the branch $|1, m\rangle_{p}^{-}$,

$$
m_{p}=\sum_{m^{\prime}=-S-(1 / 2)}^{S+(1 / 2)}\left|C_{m^{\prime}, m}^{-}\right|^{2} m^{\prime}+\sum_{m^{\prime}=-S+(1 / 2)}^{S-(1 / 2)}\left|C_{m^{\prime}, m}^{+}\right|^{2} m^{\prime},
$$

and $m_{p}=m$ when $B_{2}=B_{4}=0$.

According to Eqs. (B1) and (B2), the validity of the perturbation requires that

$$
\frac{H_{j i}^{\prime}}{E_{i}-E_{j}} \ll 1
$$

for arbitrary $i, j \in\left\{|0, m\rangle,|1, m\rangle^{-}\right\}$. By using Eqs. (B3) and (B4), one can estimate that $H_{j i}^{\prime}$ can be as large as $B_{2} S^{2}$ and $B_{4} S^{4}$ when $m \sim 0$ and $E_{i}-E_{j}$ can be as small as $D$ when $m \sim 0$. Therefore, the perturbation only applies for $B_{2} \ll D / S^{2}$ and $B_{4} \ll D / S^{4}$, which are also reasonable values for realistic $\mathrm{Mn}_{12}$ ac molecules (Table 4.1 of Ref. 19).

\section{Pauli rate equations}

When connected to the leads, the eigenstates of $H_{\mathrm{SMM}}$ can transit to each other by exchanging electrons with the lead. In the weak-coupling regime and when neutral and charged states are nearly degenerate, the sequential tunneling is dominant. The transitions are well described by the Pauli rate equations of a reduced density matrix spanned by the eigenstates of SMM. In this approach, Born approximation and Markoff approximation are employed and $H_{T}$ is treated by perturbation up to the second order. ${ }^{67}$ (Please refer to Appendix A for details.) The rate equation can be expressed in a compact form

$$
\partial_{t} P_{i}=\sum_{j} R_{i j} P_{j},
$$

where $0 \leq P_{i} \leq 1$ are the probability to find the state $i$. In this work, $i$ or $j$ belongs to the $2 S+1=21$ states from the branch $|0, m\rangle_{p}$ and $2\left(S+\frac{1}{2}\right)+1=22$ states from the branch $|1, m\rangle_{p}^{-}$, the off-diagonal and diagonal terms of the coefficient matrix are given by

$$
R_{i \neq j}=\sum_{\alpha \sigma} R_{j \rightarrow i}^{\alpha \sigma}, \quad R_{i i}=-\sum_{j \neq i} \sum_{\alpha \sigma} R_{i \rightarrow j}^{\alpha \sigma},
$$

where

$$
\begin{aligned}
R_{i \rightarrow j}^{\alpha \uparrow}= & \Gamma\left\{| \langle i | d _ { \uparrow } | j \rangle | ^ { 2 } \left[\cos ^{2}(\theta / 2) f\left(E_{j}-E_{i}-\mu_{\alpha}^{+}\right)+\sin ^{2}(\theta / 2) f\left(E_{j}\right.\right.\right. \\
& \left.\left.-E_{i}-\mu_{\alpha}^{-}\right)\right]+\left|\left\langle j\left|d_{\uparrow}\right| i\right\rangle\right|^{2}\left[\cos ^{2}(\theta / 2) f\left(E_{i}-E_{j}+\mu_{\alpha}^{+}\right)\right. \\
& \left.\left.+\sin ^{2}(\theta / 2) f\left(E_{i}-E_{j}+\mu_{\alpha}^{-}\right)\right]\right\}
\end{aligned}
$$

where the Fermi distribution $f(x)=1 /\left[\exp \left(x / k_{B} T\right)+1\right]$ is spin resolved. The coupling between LUMO and the $\alpha$ lead is assumed to be a constant parameter $\Gamma_{\alpha}^{\tau}=2 \pi \Sigma_{k}\left|V_{k \alpha}\right|^{2} \delta\left(\omega-\epsilon_{k \alpha}\right)=\Gamma$ for nonmagnetic lead. One just replaces $\uparrow$ by $\downarrow$ and exchanges + and - to obtain $R_{i \rightarrow j}^{\alpha \downarrow}$. Because the drain lead is nonmagnetic and not subjected to the spin bias, one can assume $\mu_{D}^{+--}=\mu_{D}^{\uparrow / \downarrow}$ for simplicity. For the source, $\mu_{S}^{+/-}=\mu_{S}^{\uparrow / \downarrow}$ only when $\theta=0$.

All the physical quantities can be expressed in terms of $P_{i}$, such as LUMO occupation $\sum_{i} n_{i} P_{i}$, the SMM magnetization $\sum_{i} m_{p}^{i} P_{i}$, and the $\sigma$ current flowing from the $\alpha$ lead to SMM

$$
I_{\alpha}^{\sigma}=-e \sum_{i j}\left(n_{i}-n_{j}\right) R_{j \rightarrow i}^{\alpha \sigma} P_{j},
$$

where $n_{i}$ and $m_{p}^{i}$ correspond to the $n$ and $m_{p}$ in the state $i \equiv|n, m\rangle_{p}^{\nu}$.

Notice that since we consider only the branches $|0, m\rangle$ and $|1, m\rangle^{-}$, the terms such as $\left\langle i\left|d_{\sigma}\right| k\right\rangle\left\langle j\left|d_{\sigma}^{\dagger}\right| i\right\rangle$ in the general formalism for sequential tunneling ${ }^{60}$ lead to $k=j$, which remove the off-diagonal terms $P_{i \neq j}$ from the equation of motion for $P_{i}$ and from the current formula, and lead to the Pauli-type rate equations of only diagonal terms. (Please refer to Appendix A for details.)

Although the above Pauli rate equations formalism is widely employed for the molecules weakly coupled to the electrodes, ${ }^{25,27,36-41}$ its validity in this work deserves some discussion. In the weakly coupled regime, i.e., the leadmolecule coupling $\Gamma$ is so small that between two consecutive electron tunnelings $(\sim 2 / \Gamma)$, there is enough time for the molecule to relax to the eigenstates of $H_{\mathrm{SMM}}$. For example, suppose the molecule is originally at the state $\left|0, m-\frac{1}{2}\right\rangle \equiv|0\rangle_{\mathrm{LUMO}} \otimes\left|m-\frac{1}{2}\right\rangle_{\mathrm{GS}}$. The molecule will transit to the state $|\uparrow\rangle_{\text {LUMO }} \otimes\left|m-\frac{1}{2}\right\rangle_{\mathrm{GS}}$ after a spin-up electron tunnels in. Note that $|\uparrow\rangle_{\text {LUMO }} \otimes\left|m-\frac{1}{2}\right\rangle_{\mathrm{GS}}$ is not an eigenstate of $H_{\mathrm{SMM}}$; it will relax to the eigenstate $|1, m\rangle^{-} \equiv \alpha_{m}^{-}|\downarrow\rangle_{\text {LUMO }}$ $\otimes\left|m+\frac{1}{2}\right\rangle_{\mathrm{GS}}+\beta_{m}^{-}|\uparrow\rangle_{\mathrm{LUMO}} \otimes\left|m-\frac{1}{2}\right\rangle_{\mathrm{GS}}$. The time scale of this relaxation is $\sim 1 / J$, since it is the term $-J \mathbf{S} \cdot \mathbf{S}$ that couples the states $|\downarrow\rangle_{\mathrm{LUMO}} \otimes\left|m+\frac{1}{2}\right\rangle_{\mathrm{GS}}$ and $|\uparrow\rangle_{\mathrm{LUMO}} \otimes\left|m-\frac{1}{2}\right\rangle_{\mathrm{GS}}$. To make sure that the relaxation happens before the next electron tunneling event, $J \gg \Gamma$ must be satisfied. Therefore, in this work we use $J=3.92 \mathrm{meV}$ from the first-principles calculation ${ }^{27,61}$ and $\Gamma \sim 0.01 \mathrm{meV}$.

\section{MAGNETIZATION REVERSAL AND DETECTION USING SPIN BIAS}

In this section, we will present the numerical results when $\theta=0$ and $B_{2}=B_{4}=0$. The cases when $B_{2}, B_{4} \ll D$ and $\theta \neq 0$ will be presented in Secs. V and VI, respectively. We will show both cases bring no qualitative change to the results presented in this section. When $\theta=0$ and $B_{2}=B_{4}=0$, for convenience, the notations become $\mu_{S}^{ \pm} \rightarrow \mu_{S}^{\uparrow / \downarrow}$ and $|n, m\rangle_{p}^{\nu} \rightarrow|n, m\rangle^{\nu}$.

We will first introduce the magnetization reversal induced by spin bias including hysteresis loops as a function of spin bias in Sec. IV A and avalanche dynamics near the reversal point in Sec. IV B. Finally, we will discuss the nondestructive measurement to the giant spin orientation using the spin bias in Sec. IV C.

As discussed in Sec. III B, our numerical simulations are based on the Pauli rate equations for the 43 states of 
branches $|0, m\rangle$ and $|1, m\rangle^{-}$. The parameters are already given in Sec. III B. We numerically solve Eq. (16) using RungeKutta method with the relative error smaller than $10^{-10}$. The steady solutions are obtained by relaxing the equations until for each state, its relative probability change with respect to the last iteration step is less than $10^{-7}$.

We have checked the results for total 84 states. For the energy scale of this work, the higher two branches $|1, m\rangle^{+}$ and $|2, m\rangle$ are hardly occupied. Therefore, the abandonment of these two branches is justified.

\section{A. Gate voltage tunable magnetic hysteresis loops}

When $\theta=0$ and $B_{2}=B_{4}=0$, the rate equations expressed by Eq. (16) indicate that, besides the selection rules $\left|n-n^{\prime}\right|=1$ and $\left|m-m^{\prime}\right|=\frac{1}{2}$, to trigger a transition $|0, m\rangle \rightarrow|1, m+\sigma\rangle^{-}$that adds a $\sigma$ electron onto LUMO, two conditions are required:

(C1) Energetic requirement must be satisfied so that $\mu_{\alpha}^{\sigma}>E_{|1, m+\sigma\rangle^{-}}-E_{|0, m\rangle}$.

(C2) The state $|0, m\rangle$ should be occupied.

Similarly, to trigger a transition $|1, m+\sigma\rangle^{-} \rightarrow|0, m\rangle$ that removes a $\sigma$ electron from LUMO, it is required that:

(C3) Energetic requirement must be satisfied so that $\mu_{\alpha}^{\sigma}<E_{|1, m+\sigma\rangle^{-}}-E_{|0, m\rangle}$.

(C4) The state $|1, m+\sigma\rangle$ should be occupied.

To generate the charging-discharging sequence from $|1,-21 / 2\rangle$ through $|1,21 / 2\rangle$ shown in Fig. 2 , the energetic requirements $(\mathrm{C} 1)$ must be satisfied for each of them. We list all the 42 transition energies $E_{|1, m \pm 1 / 2\rangle^{-}}-E_{|0, m\rangle}$ in Fig. 3. Note that for the present model and parameters the low and high bounds of the entire spectrum happen to be $E_{|1, \pm 19 / 2\rangle^{-}}-E_{|0, \pm 10\rangle}$ and $E_{|1, \pm 5 / 2\rangle^{-}}-E_{|0, \pm 2\rangle}$, respectively. Note that the highest and the lowest transition energies may differ from sample to sample, but the following qualitative results are unaffected. The reversal $S_{z}=-10 \rightarrow 10$ requires $V$ large enough to satisfy

$$
\begin{aligned}
& \mu_{S}^{\uparrow}=\frac{V}{2}>E_{|1, \pm 19 / 2\rangle^{-}}-E_{|0, \pm 10\rangle}, \\
& \mu_{S}^{\downarrow}=-\frac{V}{2}<E_{|1, \pm 5 / 2\rangle^{-}}-E_{|0, \pm 2\rangle},
\end{aligned}
$$

which thereby defines a threshold voltage ${ }^{37}$ or activation energy. ${ }^{41}$ Figure 3 indicates clearly that the activation energy is determined not only by the highest $E_{|1, \pm 19 / 2\rangle^{-}}-E_{|0, \pm 10\rangle}, 37,41$ but also by the lowest $E_{|1, \pm 5 / 2\rangle^{-}}-E_{|0, \pm 2\rangle}$. More importantly, as shown in Fig. 3 the entire spectra of all 42 transition energies can be shifted with respect to $\mu_{S}^{\uparrow / \downarrow}$ by tuning $V_{g}$, which means that the activation energy is tunable by the gate voltage. The activation energy as a function of $V_{g}$ is shown in Fig. 4(b). The activation energy can be minimized when the center of the entire spectrum is aligned with 0 , which is about $V_{g}=-18.8 \mathrm{mV}$ for the present parameters. When $V_{g}<-18.8$, e.g., $V_{g}=-19.6$, the activation energy is determined by $E_{|1, \pm 19 / 2\rangle^{-}}-E_{|0, \pm 10\rangle}$. When $V_{g}>-18.8$, e.g., $V_{g}=-17.2$, the activation energy is determined by $E_{|1, \pm 5 / 2\rangle^{-}}-E_{|0, \pm 2\rangle}$. Using similar analysis, one knows that the reversal $S_{z}=10 \rightarrow-10$ requires

$$
\begin{gathered}
\mu_{S}^{\downarrow}=-\frac{V}{2}>E_{|1, \pm 19 / 2\rangle^{-}}-E_{|0, \pm 10\rangle}, \\
\mu_{S}^{\uparrow}=\frac{V}{2}<E_{|1, \pm 5 / 2\rangle^{-}}-E_{|0, \pm 2\rangle} .
\end{gathered}
$$

As a result, the magnetization when sweeping $V$ back and forth must exhibit a hysteresis loop. As shown in Fig. 4(a), the hysteresis loop varies with $V_{g}$ because the activation energy is tunable by the gate voltage. When considering the broadening of the Fermi surface at higher temperatures, thermal fluctuation will activate the magnetic reversal before $V$ reaches exactly the required activation energy. As a consequence, the magnetic hysteresis loop shrinks when the temperature increases [Fig. 4(c)].

Note that the existence of the activation energy and its tunability to the gate voltage only results from that the spectrum of all the transition energies has a finite width and the spectrum must has a finite width because of the anisotropy. Therefore, although in the reality the highest and lowest transition energies may differ from sample to sample, the above results are qualitatively unaffected.

\section{B. Avalanche dynamics at magnetic reversal point}

We investigate the dynamics at the reversal point marked by the triangle in Fig. 4(a). On the left side of this point, the activation energy is determined by $E_{|1, \pm 19 / 2\rangle^{-}}-E_{|0, \pm 10\rangle}$ and $V$ is smaller than $2\left(E_{|1,-19 / 2\rangle^{-}}-E_{|0,-10\rangle}\right)$ while larger than the rest 40 transition energies. Therefore, the reversal is blocked at the state $|0,-10\rangle$ only because $|1,-19 / 2\rangle^{-}$cannot be occupied. Once $|1,-19 / 2\rangle^{-}$is occupied when $V$ exceeds $2\left(E_{|1,-19 / 2\rangle^{-}}-E_{|0,-10\rangle}\right)$, an avalanche of the rest 40 transitions will be triggered. In Figs. 5 and 6, we demonstrate the numerical simulation of this avalanche by showing the timedependent probabilities for the states of the branches $|0, m\rangle$ and $|1, m\rangle^{-}$when $V_{g}=-18.8 \mathrm{mV}$ and $V=1.8 \mathrm{meV}$ $>2\left(E_{|1,-19 / 2\rangle^{-}}-E_{|0,-10\rangle}\right)$. As we see, all the intermediate states between $|0,-10\rangle$ and $|1,21 / 2\rangle$ show a clear time sequence. Each of them is first occupied, then reaches a maximum, and finally decays to zero. The time scale of the avalanche process can be estimated by $\sim 300 \frac{\hbar}{\Gamma}$, only $\sim 6$ ns if we choose the experiment fitting parameter ${ }^{21} \Gamma=8 \mathrm{GHz}$ $\approx 0.033 \mathrm{meV}$.

\section{Nondestructive detection to giant spin orientation}

A scheme to read out the SMM magnetization has been proposed by Timm and Elste, ${ }^{37}$ in which however the readout is accompanied by the decay of SMM magnetization. Here we propose a detection scheme, in which the giant spin of a SMM has already been prepared to be at either $S$ or $-S$ ground state. Our goal is to detect at which of the two orientations the giant spin points, and most importantly, without destroying the giant spin orientation by means of spin bias. ${ }^{55}$ We have shown in Fig. 5 that in the presence of only one lead only a burst of pure spin current can flow only during 


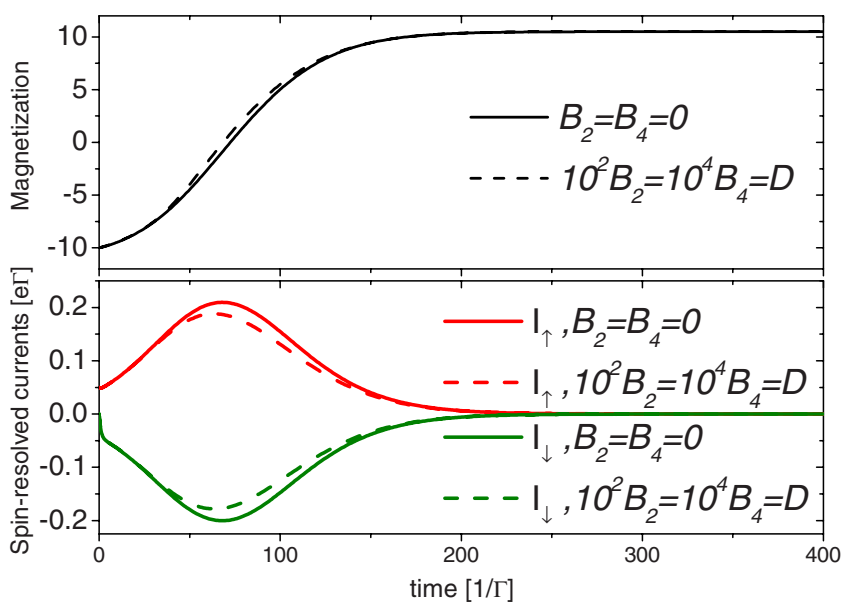

FIG. 5. (Color online) The time-dependent magnetization and the spin-resolved currents during the magnetic reversal. Solid and dashed lines represent the cases in the absence and the presence of a weak transverse anisotropy, respectively. The initial state is $P_{|0,-10\rangle}=1 \quad$ and $\quad P_{i \neq|0,-10\rangle}=0 . \quad T=0.01 \mathrm{~K}, \quad V_{g}=-18.8 \mathrm{mV}$, $V=1.8 \mathrm{meV}$ [the triangle in Fig. 4(a)]. Other parameters are given in Sec. III B. The positive sign of current stands for flowing from source to SMM and negative for from SMM to source.

the magnetic reversal, i.e., one lead is not enough to maintain a steady current. Thus, we introduce two leads for this detection as shown in Fig. 7(b). A small spin bias $V=0.05 \mathrm{meV}$ is applied to only the source lead so that $\mu_{S}^{\uparrow / \downarrow}= \pm \frac{V}{2}$, while the Fermi level for the drain lead $\mu_{D}^{\downarrow / \uparrow}$ is set at 0 . By tuning the gate voltage $V_{g}$, the Fermi levels of leads $\mu_{S / D}^{\uparrow / \downarrow}$ can be located aligned with the two degenerate transition energies $E_{|1, \pm 21 / 2\rangle^{-}}-E_{|0, \pm 10\rangle}$. Taking Figs. 7(c1)-7(c4), for example, the giant spin orientation is initiated at
$S_{z}=-10$, i.e., either $|1,-21 / 2\rangle$ or $|0,-10\rangle$ is likely to be occupied. On the left side of Figs. 7(c2)-7(c4), the molecule is at the state $|0,-10\rangle$; on the right side of Figs. 7(c2)-7(c4), the molecule is at $|1,-21 / 2\rangle$. Notice that the transition between them involves only $\downarrow$ electron. As shown in Fig. 7(b), when

$$
\mu_{S}^{\downarrow}<E_{|1, \pm 21 / 2\rangle^{-}}-E_{|0, \pm 10\rangle} \leq \mu_{D}^{\downarrow},
$$

a spin-down electron can be injected from the drain lead, inducing the transition $|0,-10\rangle \rightarrow|1,-21 / 2\rangle$, then leaks to the source lead, making the molecule recover to the state $|0,-10\rangle$. Such process repeats continuously, leading to a steady fully polarized spin-down electric current flowing from the drain to the source lead. Since we define the direction of current from source to drain as positive, the spin- $\downarrow$ current is negative, as shown by the negative peak in Fig. 7(c2).

For the same situation shown in Fig. 7(b), if the giant spin is oriented along $S_{z}=10$ as shown in Figs. 7(d1)-7(d4), only the transition between $|1,21 / 2\rangle$ and $|0,10\rangle$ is possible, which will generate a positive $\uparrow$ current from source to drain. Because of the degeneracy of $E_{|1,21 / 2\rangle^{-}}-E_{|0,10\rangle}$ and $E_{|1,-21 / 2\rangle^{-}}-E_{|0,-10\rangle}$, the spin polarization and flowing direction of the steady current depend only on the giant spin orientation.

Notice that the detection does not change the giant spin orientation from initiated $S_{z}= \pm 10$ to other values. Specifically, in order to destroy the state initiated $S_{z}=-10$, the state $|0,-10\rangle$ has to transit to $|1,-19 / 2\rangle^{-}$first, which requires two conditions simultaneously: (1) $\mu_{S / D}^{\uparrow}>E_{|1,-19 / 2\rangle^{-}}-E_{|0,-10\rangle}$ and (2) $|0,-10\rangle$ is occupied. According to Fig. 3, to make $\mu_{S / D}^{\uparrow}>E_{|1,-19 / 2\rangle^{-}}-E_{|0,-10\rangle}, V_{g}$ must be $>-18.8 \mathrm{mV}$. However, when $V_{g}>-18.8$, only $|1,-21 / 2\rangle$ is allowed to be and
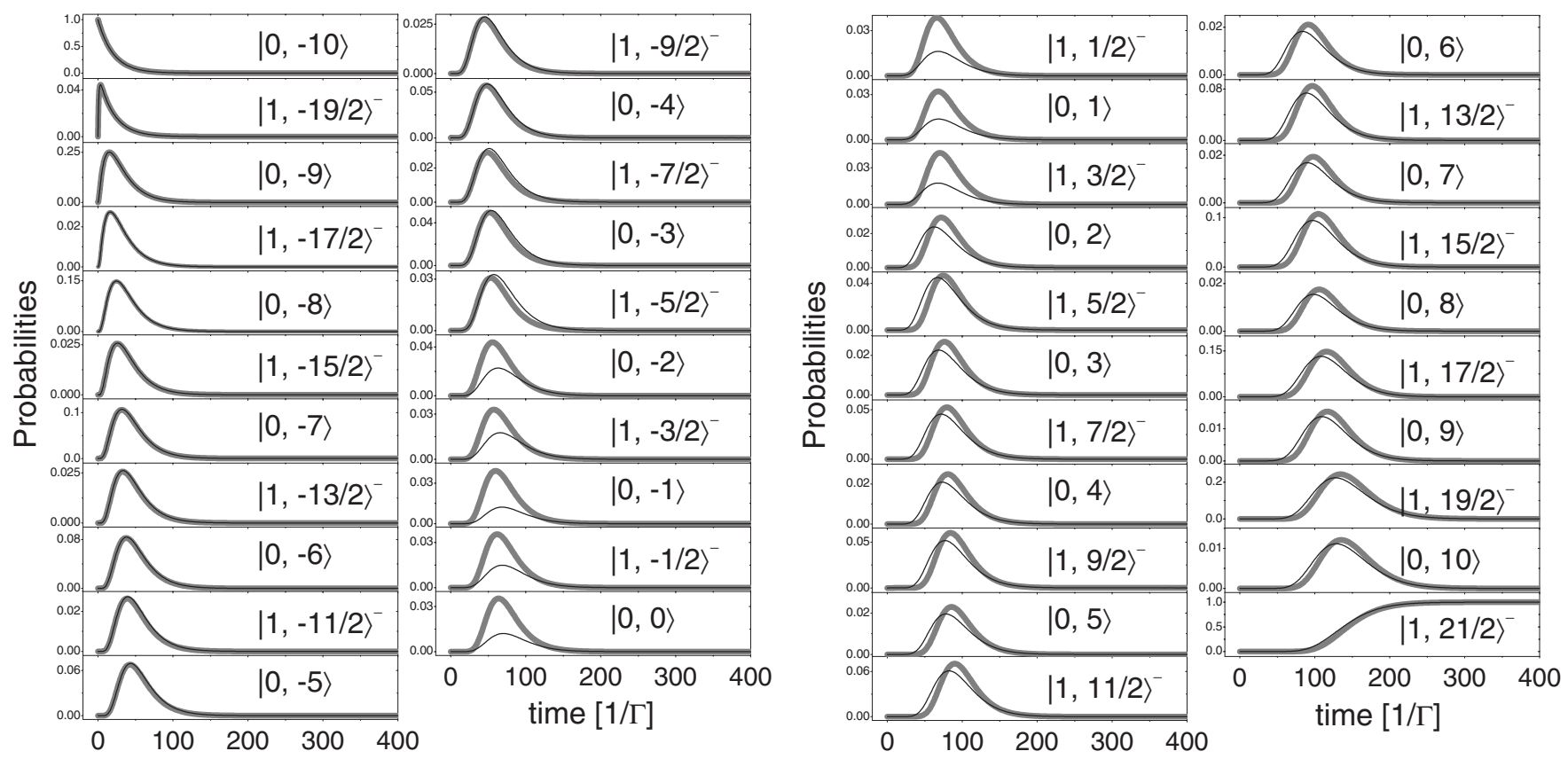

FIG. 6. During the magnetic reversal, the time-dependent probabilities of the branches $|0, m\rangle$ and $|1, m\rangle^{-}$. Thick and thin lines represent the cases when $B_{2}=B_{4}=0$ and $10^{2} B_{2}=10^{4} B_{4}=D$, respectively. Note that the states from $|0,-2\rangle$ through $|0,2\rangle$ are explicitly reshaped by the $B_{2}$ and $B_{4}$. All the parameters are the same as Fig. 5 . 


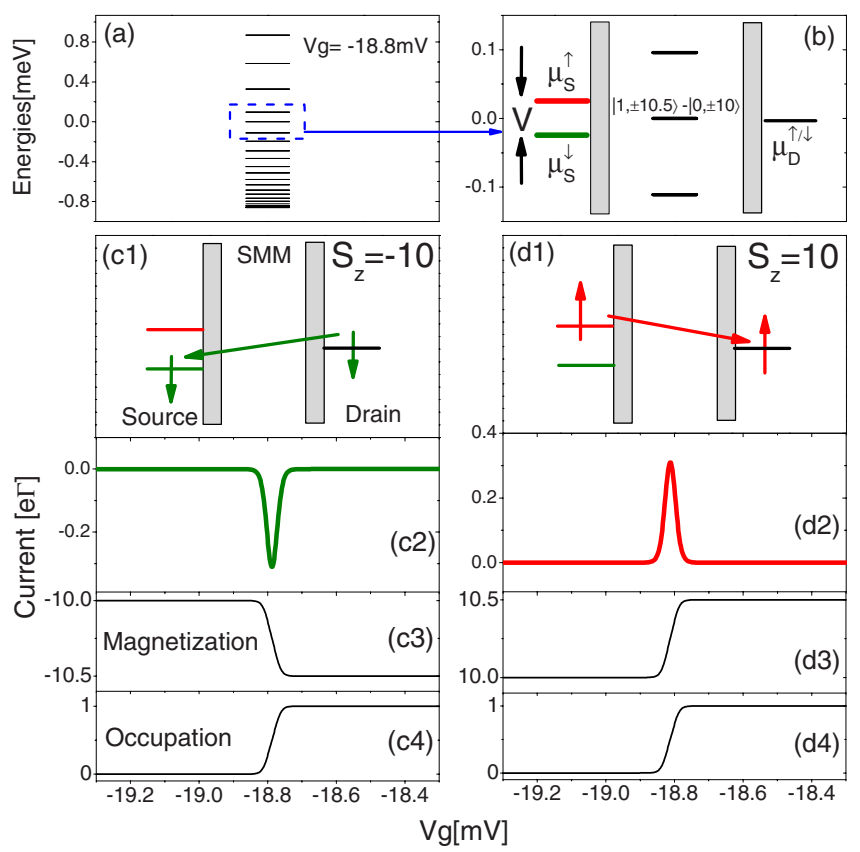

FIG. 7. (Color online) Nondestructive detection to the giant spin orientation. (a) Transition energies $E_{|1, m \pm 1 / 2\rangle^{-}}-E_{|0, m\rangle}$, when $V_{g}=$ $-18.8 \mathrm{mV}$. (b) Zoom-in of (a) near $E_{|1, \pm 21 / 2\rangle}-E_{|0, \pm 10\rangle}$. By tuning the gate voltage $V_{g}$, the Fermi levels of leads $\mu_{S / D}^{\uparrow /}$ can be located aligned with the two degenerate transition energies $E_{|1, \pm 21 / 2\rangle^{-}}$ $-E_{|0, \pm 10\rangle} \cdot[(\mathrm{c} 1)$ and (d1)] Schematics of how fully polarized electric current flows when $S_{z}= \pm 10$. [(c2)-(c4) and (d2)-(d4)] For $S_{z}$ $= \pm 10$, numerical results of source-to-drain current, SMM magnetization, and LUMO occupation as functions of $V_{g}$ in the presence of a small $V=0.05 \mathrm{meV} . T=0.1 \mathrm{~K}$. Other parameters are given in Sec. III B.

fully occupied, instead of $|0,-10\rangle$, as shown by the occupation and magnetization in Figs. 7(c3) and 7(c4). Therefore, our detection scheme is nondestructive.

\section{EFFECT OF TRANSVERSE ANISOTROPY}

In this section, we will show that the small transverse anisotropy only weakly affects the three main results presented in Sec. IV and brings no qualitative change. The transverse anisotropy leads to two main corrections of the SMM eigenstates. The first is the quantitative correction to the energy and the projection of the magnetization along $z$-axis $m_{p}$ [defined by Eqs. (13) and (14)]. The second is the weak violation to the spin selection rules ${ }^{25}$ according to Eq. (12) so that transitions between states $\left|m_{p}-m_{p}^{\prime}\right|>1 / 2$ now are possible.

In Fig. 8, we show the energy as a function of the projection of magnetization along $z$-axis $m_{p}$ for each SMM states, when $B_{2}=10^{-2} \mathrm{D}$ and $B_{4}=10^{-4} \mathrm{D}$. The case when $B_{2}=B_{4}=0$ is also plotted for comparison. As we see, the changes in energy and $m_{p}$ are ignorably small for those states with $\left|m_{p}\right|$ $\sim S$. However, the states with $\left|m_{p}\right| \sim 0$ are greatly reshaped and in some extent mixed together.

The influence of this mixture on the reversal dynamics is demonstrated by the thin lines in Fig. 6. Let us

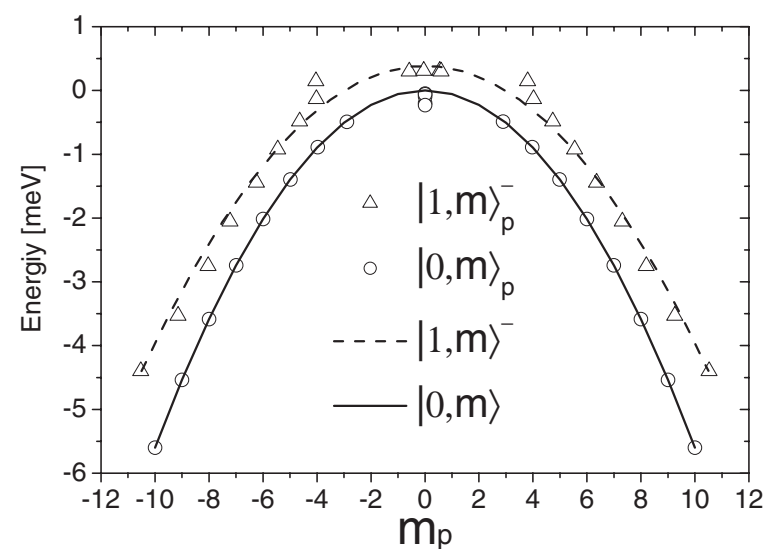

FIG. 8. The energies of the perturbed branches $|0, m\rangle_{p}$ and $|1, m\rangle_{p}^{-}$as a function of perturbed projection $m_{p}$. Circle and triangle: $B_{2}=10^{-2} D, B_{4}=10^{-4} D$. Solid and dashed lines: $B_{2}=B_{4}=0$. Other parameters are the same as Fig. 2.

focus on the subfigures from $|0,-2\rangle_{p}$ through $|0,2\rangle_{p}$. Due to the mixture of $|0,-2\rangle_{p}$ and $|0,2\rangle_{p}$, once SMM evolves to the state $|0,-2\rangle_{p}$, there is certain probability that SMM continues evolving to $|0,2\rangle_{p}$ directly without through $|1,-3 / 2\rangle_{p} \rightarrow|0,-1\rangle_{p}^{-} \rightarrow|1,-1 / 2\rangle_{p}^{-} \rightarrow|0,0\rangle_{p}$ $\rightarrow|1,1 / 2\rangle_{p}^{-} \rightarrow|1,1\rangle_{p} \rightarrow|1,3 / 2\rangle_{p}^{-} \rightarrow|0,2\rangle_{p}$. As a result, the probabilities of these intermediate states become smaller compared with those when $B_{2}=B_{4}=0$. Accordingly, the reversal time is also slightly shortened, as shown by the dashed lines in Fig. 5.

The hysteresis loops in the presence of the transverse anisotropy are shown in Fig. 9. The hysteresis loop when $V_{g}=-19.6$ is not changed because it is determined by $E_{|1, \pm 19 / 2\rangle_{p}^{-}}-E_{|0, \pm 10\rangle_{p}}$, where both $E_{|1, \pm 19 / 2\rangle_{p}^{-}}$and $E_{|0, \pm 10\rangle_{p}}$ are hardly affected by the transverse anisotropy. On the other side, the hysteresis loop when $V_{g}=-17.2$ is slightly modified
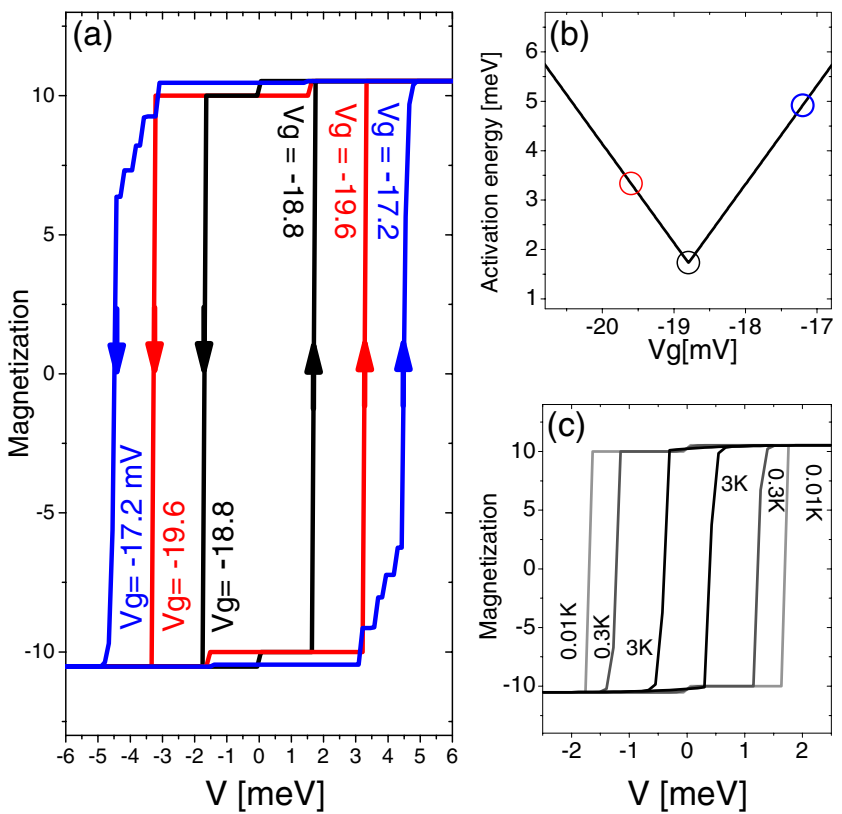

FIG. 9. (Color online) The same as Fig. 4 except $B_{2}=10^{-2} D$ and $B_{4}=10^{-4} \mathrm{D}$. 

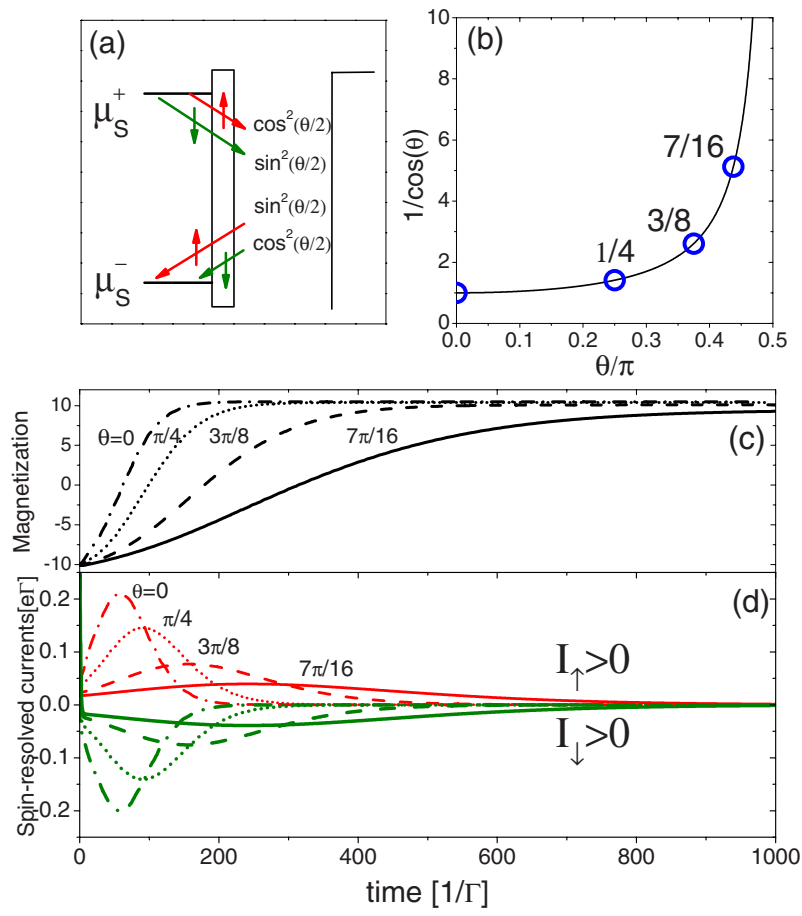

FIG. 10. (Color online) (a) In the presence of an angle $\theta$ between the easy axis of SMM and the spin-quantization direction of the lead, there are four possible injection and leakage processes marked by the arrows. $\cos ^{2}(\theta / 2)$ or $\sin ^{2}(\theta / 2)$ indicates the relative probability of each process. (b) The reversal time will be prolonged by $1 / \cos (\theta)$. [(c) and (d)] Magnetization and spin-resolved currents for the same situation shown in Fig. 5 for different $\theta$.

because it is determined by $E_{|1, \pm 5 / 2\rangle_{p}^{-}}-E_{|0, \pm 2\rangle_{p}}$, while $E_{|1, \pm 5 / 2\rangle_{p}^{-}}$and $E_{|0,2\rangle_{p}}$ are reshaped by the transverse anisotropy.

According to Fig. 8, the energies of $|0, \pm 10\rangle_{p}$ and $|1, \pm 21 / 2\rangle_{p}^{-}$are barely affected by weak $B_{2}$ and $B_{4}$. Therefore, the measurements discussed in Sec. IV $C$ are not affected noticeably. Although the spin selection rules now allow transitions between $|1,21 / 2\rangle_{p}^{-}$and states other that $|0,10\rangle_{p}$, e.g., $|1,21 / 2\rangle_{p}^{-} \leftrightarrow|0,9\rangle_{p}$, these kind of transitions have probabilities of order of $\left(B_{2} / D\right)^{2} \sim 10^{-4}$, thus is hard to be measured.

\section{NONCOLLINEAR CASE}

In this section, we will analyze the influence to the three main results presented in Sec. IV when considering an angle $\theta$ between the easy axis of the SMM and the spinquantization direction of the source lead. Because we have shown that the weak transverse anisotropy brings ignorable effect in Sec. V, we only consider $B_{2}=B_{4}=0$ in this section.

\section{A. Reversal dynamics $\theta \neq 0$}

When $\theta \neq 0$, the $+(-)$ electrons in the source lead can be injected into SMM as $\uparrow(\downarrow)$ or $\uparrow(\downarrow)$ electrons, with probabilities of $\cos ^{2} \frac{\theta}{2}$ and $\sin ^{2} \frac{\theta}{2}$, respectively. This will prolong the reversal time. We will illustrate it by considering the same situation of Sec. IV B. As shown in Fig. 10(a), when $\uparrow$ electrons are injected from $\mu_{S}^{+}$with the tunneling rate $\propto \cos ^{2} \frac{\theta}{2}$, they are also leaking to $\mu_{S}^{-}$with the tunneling rate $\propto \sin ^{2} \frac{\theta}{2}$. From the viewpoint of SMM, the $\uparrow$ electrons are injected from the lead at an equivalent tunneling rate $\propto \cos ^{2} \frac{\theta}{2}-\sin ^{2} \frac{\theta}{2}=\cos \theta$. Similarly, an extra ratio $\cos \theta$ is also subjected to the $\downarrow$ electron leaking to the lead. As a result, the reversal time will $\propto 1 / \cos \theta$, as shown by Figs. 10(c) and 10(d). As shown by Fig. 10(b), $\theta$ should be at least $>0.45 \pi$ to increase the reversal time by 1 order. In other words, the reversal time will not be prolonged dramatically unless $\theta$ is very close to $\pi / 2$. Of course, the reversal will fail when $\theta=\pi / 2$, i.e., when the spin-quantization direction of the lead is perpendicular to the easy axis of SMM. Besides, as shown by Fig. 10(d), during the reversal, there is still a pure spin current flowing even in the presence of $\theta \neq 0$, although $\theta$ will introduce the extra injection of $\downarrow$ electrons from $\mu_{S}^{+}$and the extra leakage of $\uparrow$ electrons to $\mu_{S}^{-}$. This is because these two effects will cancel with each other since both of them are proportional to $\sin ^{2} \frac{\theta}{2}$.

\section{B. Nondestructive detection $\boldsymbol{\theta} \neq 0$}

As for the nondestructive measurement in Sec. IV C, the qualitative nature that spin-up (-down) current is favored when $S_{z}=S(-S)$ is irrelevant to $\theta$. Besides, our numerical results show that the measurement is still nondestructive. However, in the presence of $\theta \neq 0$, both $\uparrow$ and $\downarrow$ electrons can tunnel via the Fermi level $\mu_{S}^{+}$and $\mu_{S}^{-}$. As a result, the current can flow along both directions, different from the $\theta=0$ results shown in Figs. 7(c2) and 7(d2).

We will use the case $S_{z}=-S$ to illustrate this difference, as shown by Figs. 11(c1)-11(c3). When $S_{z}=-S$, SMM still favors $\downarrow$ current to tunnel through it via the transition energy $E_{|1, \pm 21 / 2\rangle^{-}}-E_{|0, \pm 10\rangle}$. As shown by Fig. 11(a), when

$$
\mu_{S}^{-}<E_{|1, \pm 21 / 2\rangle^{-}}-E_{|0, \pm 10\rangle}<\mu_{D}^{\uparrow / \downarrow}<\mu_{S}^{+},
$$

current will favor tunneling from drain to source with a relative probability $\propto \cos ^{2}(\theta / 2)$ and this corresponds to the negative current on the left of Figs. 11(c1)-11(c3); while as shown by Fig. 11(b), when

$$
\mu_{S}^{-}<\mu_{D}^{\uparrow / \downarrow}<E_{|1, \pm 21 / 2\rangle^{-}}-E_{|0, \pm 10\rangle}<\mu_{S}^{+},
$$

current will favor tunneling from source to drain with a relative probability $\propto \sin ^{2}(\theta / 2)$ and this corresponds to the positive current on the right of Figs. 11(c1)-11(c3).

The case for $S_{z}=S$ is similar, except that only $\uparrow$ current is flowing, and the relative ratio between the magnitude of the negative and positive currents becomes $\sin ^{2} \frac{\theta}{2} / \cos ^{2} \frac{\theta}{2}$. So one can still use this difference to distinguish the orientation of the giant spin, unless $\theta$ is very close to $\pi / 2$. When $\theta=\pi / 2$, the negative and positive currents are the same, as shown by Figs. 11(c3) and 11(d3). This is reasonable result because when the spin orientation of the source lead is perpendicular to the easy axis of SMM, one should have symmetric results for $\uparrow$ and $\downarrow$.

\section{Summary for $\theta \neq 0$}

We briefly summarize the influences of $\theta \neq 0$. Since $\theta$ does not change the energy of many-body states, the steady- 


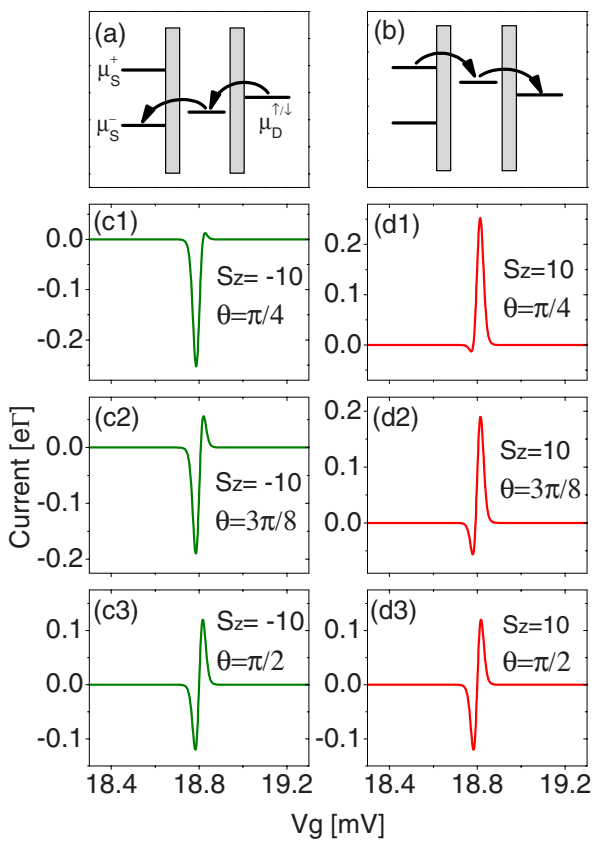

FIG. 11. (Color online) (a) Current tends to flow from drain to source when $E_{|1, \pm 21 / 2\rangle^{-}}-E_{|0, \pm 10\rangle}$ is between $\mu_{S}^{-}$and $\mu_{D}^{\uparrow / \downarrow}$. (b) Current tends to flow from source to drain when $E_{|1, \pm 21 / 2\rangle^{-}}-E_{|0, \pm 10\rangle}$ is between $\mu_{S}^{+}$and $\mu_{D}^{\uparrow / \downarrow}$. $[(\mathrm{c} 1)-(\mathrm{c} 3)]$ The same measurement scheme as Fig. 7(c2) except for $\theta \neq 0$. [(d1)-(d3)] The same measurement scheme as Fig. 7(d2) except for $\theta \neq 0$.

state solutions of hysteresis loops shown in Fig. 4 are not affected except $\theta \sim \pi / 2$. It will prolong the reversal time by $1 / \cos \theta$, as shown in Fig. 10. There is still a pure spin current flowing during the reversal when $\theta \neq 0$. The nondestructive measurement shown in Fig. 7 is still nondestructive when $\theta \neq 0$. However, the measurement signals will be reshaped as shown in Fig. 11. Fortunately, one can still employ the relative ratio between current flowing along opposite directions to distinguish the orientation of the giant spin unless $\theta \sim \pi / 2$.

\section{SUMMARY AND DISCUSSION}

Before ending this paper, we compare the advantages of the previous works on current-induced reversal of SMM magnetization to our proposal. For the setup with one nonmagnetic and one ferromagnetic lead ${ }^{37}$ or the setup with two magnetic leads that are not fully polarized, ${ }^{41}$ electrons injected from one lead can leak to the other lead, while electrons hopping to one lead can be refilled by electrons from the other lead, as shown in Figs. 12(a) and 12(b). The leakage and refilling reduce the efficiency of magnetization reversal because only the excess transmitted spins contribute to the process of the magnetic reversal. ${ }^{37}$ Because no excess spin is transmitted, two parallel aligned ferromagnetic leads $^{41}$ are equivalent to two nonmagnetic leads when the charge bias is large enough to cover all the transition energies, as shown in Fig. 12(c). Moreover, the electric current induced by the charge bias between two nonmagnetic electrodes may lead to the decay of SMM magnetization. ${ }^{37}$ In
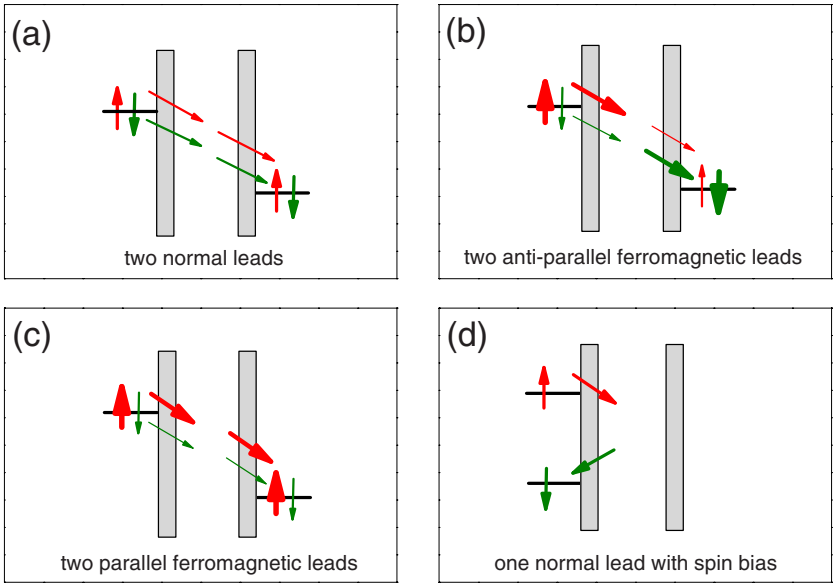

FIG. 12. (Color online) (a)-(c) Energy schemes employed by the previous authors and (d) in this work. (a) Nonmagnetic leads (Ref. 37), (b) ferromagnetic leads with antiparallel polarizations, and (c) ferromagnetic leads with parallel polarizations (Ref. 41); (d) nonmagnetic lead with spin-dependent splitting of chemical potentials.

contrast, the current-induced relaxation and low efficiency can be avoided in our one-lead model, as only pure spin current flows during the reversal and no lead-SMM electron exchange is permitted when there is no reversal occurring. Technically, the ferromagnetic lead can already be attached to the single molecule with the charge bias voltage easily exceeding the required threshold voltage, ${ }^{68}$ while large spin bias over $1 \mathrm{meV}$ still remains an experimental challenge. In conclusion, the spin bias or spin current can be applied to control and measure the magnetization of SMM efficiently.

\section{ACKNOWLEDGMENTS}

The authors thank Qian Niu, Guoxiang Huang, Ren-Bao Liu, and Rong Lü for helpful discussions. This work is supported by the Research Grant Council of Hong Kong under Grants No. HKU 7048/09P and No. HKU 10/CRF/08.

\section{APPENDIX A: THE RATE EQUATIONS}

In this appendix, we will present the deduction of the rate equations Eq. (16) following the approach introduced in Ref. 67 . Besides, we will explain why only diagonal terms of the reduced density matrix are employed for the present problem. For simplicity, only $\theta=0$ case will be addressed. The case for $\theta \neq 0$ is a straightforward generalization.

Suppose we have found the eigenstates $|n\rangle$ of $H_{\mathrm{SMM}}$. By using the completeness $\Sigma_{n}|n\rangle\langle n|=$ unity, the Hamiltonian Eqs. (4) and (7) can be rewritten as

$$
H_{\mathrm{SMM}}=\sum_{n} E_{n}|n\rangle\langle n|
$$

and

$$
H_{T}=\sum_{k, \alpha, \sigma} \sum_{n, m}\left(V_{k \alpha \sigma}\left\langle n\left|d_{\sigma}\right| m\right\rangle c_{k \alpha \sigma}^{\dagger}|n\rangle\langle m|\right)+\text { H.c. }
$$


The Liouville equation of the density matrix $\hat{\rho}$ of the entire system is given by

$$
\partial_{t} \hat{\rho}=-i[H, \hat{\rho}],
$$

where $H=H_{\mathrm{SMM}}+H_{\text {lead }}+H_{T}$. For arbitrary operator $\hat{O}$, one introduces the interaction picture $\hat{O}_{I}(t)=e^{i H_{0} t} \hat{O} e^{-i H_{0} t}$, where $H_{0}=H_{\text {SMM }}+H_{\text {lead }}$, then Eq. (A3) becomes

$$
\partial_{t} \hat{\rho}_{I}(t)=-i\left[H_{T I}(t), \hat{\rho}_{I}(t)\right] .
$$

Integrate and iterate Eq. (A4) for one time

$$
\partial_{t} \hat{\rho}_{I}(t) \approx-i\left[H_{T I}(t), \hat{\rho}_{I}(0)\right]-\int_{0}^{t} d t^{\prime}\left\{H_{T I}(t),\left[H_{T I}\left(t^{\prime}\right), \hat{\rho}_{I}\left(t^{\prime}\right)\right]\right\} .
$$

The reduced density matrix $\hat{\rho}_{M}(t)$ of SMM is obtained from $\hat{\rho}(t)$ by taking the trace over all variables of the leads, i.e., in the interaction picture,

$$
\hat{\rho}_{M I} \equiv \operatorname{Tr}_{L} \hat{\rho}_{I}
$$

Trace out the lead part in Eq. (A5)

$$
\begin{aligned}
\partial_{t} \hat{\rho}_{M I}(t)= & -i \operatorname{Tr}_{L}\left[H_{T I}(t), \hat{\rho}_{I}(0)\right] \\
& -\int_{0}^{t} d t^{\prime} \operatorname{Tr}_{L}\left\{H_{T I}(t),\left[H_{T I}\left(t^{\prime}\right), \hat{\rho}_{I}\left(t^{\prime}\right)\right]\right\} .
\end{aligned}
$$

The above equation assumed that $H_{T}$ is switched on at $t=0$. Prior to this, SMM and the leads are uncorrelated and the total density matrix is given by their direct products,

$$
\hat{\rho}(0)=\hat{\rho}_{M}(0) \otimes \hat{\rho}_{L}(0)=\hat{\rho}_{I}(0) .
$$

At this point, we follow Fano to make two key assumptions. ${ }^{69}$ The first is the Born approximation, which assumes that the leads have so many degrees of freedom that the effects of interaction with SMM dissipate away quickly and will not react back to any significant extent, so that the leads remain described by a thermal equilibrium distribution at constant temperature $\hat{\rho}_{L}(0)$ at all time

$$
\hat{\rho}_{I}(t) \rightarrow \hat{\rho}_{M I}(t) \otimes \hat{\rho}_{L}(0) . \quad(\text { Born })
$$

The second is the Markoff approximation, which assumes that due to the rapid relaxation in the leads, correlation functions of lead electrons decay on a time scale much shorter than the SMM dynamics, i.e., the leads do not have memory. This allows replacing the correlation functions by delta functions in the rates, which are convolutions of such correlation functions with the reduced density matrix in the sequentialtunneling approximation. In this context, $\partial_{t} \hat{\rho}_{M I}(t)$ depends only on its present value $\hat{\rho}_{M I}(t)$,

$$
\hat{\rho}_{M I}\left(t^{\prime}\right) \rightarrow \hat{\rho}_{M I}(t) . \quad \text { (Markoff) }
$$

Put the two approximations (A9) and (A10) into Eq. (A7),

$$
\begin{aligned}
\partial_{t} \hat{\rho}_{M I}(t)= & -i \operatorname{Tr}_{L}\left[H_{T I}(t), \hat{\rho}_{M I}(0) \hat{\rho}_{L}(0)\right] \\
& -\int_{0}^{t} d t^{\prime} \operatorname{Tr}_{L}\left\{H_{T I}(t),\left[H_{T I}\left(t^{\prime}\right), \hat{\rho}_{M I}(t) \otimes \hat{\rho}_{L}(0)\right]\right\} .
\end{aligned}
$$

What follows is straightforward calculation by putting $H_{T I}$ into Eq. (A11). The first term on the right side of Eq. (A11) vanishes because

$$
\operatorname{Tr}_{L}\left[c_{k \alpha \sigma I}^{\dagger}(t) \hat{\rho}_{L}(0)\right]=\operatorname{Tr}_{L}\left[c_{k \alpha \sigma I}(t) \hat{\rho}_{L}(0)\right]=0 .
$$

Besides, the cyclic property of the trace is used

$$
\operatorname{Tr}_{L}\left[c_{k \alpha \sigma I}^{\dagger}(t) \dot{c}_{k^{\prime} \alpha^{\prime} \sigma^{\prime} I}\left(t^{\prime}\right) \hat{\rho}_{L}(0)\right]=\operatorname{Tr}_{L}\left[c_{k \alpha \sigma I}^{\dagger}\left(t-t^{\prime}\right) c_{k^{\prime} \alpha^{\prime} \sigma^{\prime}} \hat{\rho}_{L}(0)\right] .
$$

Finally, one returns to the Schrödinger picture and arrives at the equation for the arbitrary terms $P_{i j} \equiv\left\langle i\left|\hat{\rho}_{M}\right| j\right\rangle$,

$$
\begin{aligned}
\partial_{t} P_{i j}= & -i\left(E_{i}-E_{j}\right) P_{i j} \\
& -\frac{1}{2} \sum_{i^{\prime} j^{\prime}} \sum_{\alpha \sigma} \Gamma_{\alpha}^{\sigma}\left\langle i\left|d_{\sigma}\right| j^{\prime}\right\rangle\left\langle j^{\prime}\left|d_{\sigma}^{\dagger}\right| i^{\prime}\right\rangle f\left(E_{j^{\prime}}-E_{i^{\prime}}-\mu_{\alpha}^{\sigma}\right) P_{i^{\prime} j} \\
& -\frac{1}{2} \sum_{i^{\prime} j^{\prime}} \sum_{\alpha \sigma} \Gamma_{\alpha}^{\sigma}\left\langle j^{\prime}\left|d_{\sigma}\right| i^{\prime}\right\rangle\left\langle i\left|d_{\sigma}^{\dagger}\right| j^{\prime}\right\rangle\left[f\left(E_{i^{\prime}}-E_{j^{\prime}}+\mu_{\alpha}^{\sigma}\right)\right] P_{i^{\prime} j} \\
& +\frac{1}{2} \sum_{i^{\prime} j^{\prime}} \sum_{\alpha \sigma} \Gamma_{\alpha}^{\sigma}\left\langle i\left|d_{\sigma}\right| i^{\prime}\right\rangle\left\langle j^{\prime}\left|d_{\sigma}^{\dagger}\right| j\right\rangle\left[f\left(E_{j^{\prime}}-E_{j}+\mu_{\alpha}^{\sigma}\right)\right] P_{i^{\prime} j^{\prime}} \\
& +\frac{1}{2} \sum_{i^{\prime} j^{\prime}} \sum_{\alpha \sigma} \Gamma_{\alpha}^{\sigma}\left\langle j^{\prime}\left|d_{\sigma}\right| j\right\rangle\left\langle i\left|d_{\sigma}^{\dagger}\right| i^{\prime}\right\rangle f\left(E_{j}-E_{j^{\prime}}-\mu_{\alpha}^{\sigma}\right) P_{i^{\prime} j^{\prime}} \\
& +\frac{1}{2} \sum_{i^{\prime} j^{\prime}} \sum_{\alpha \sigma} \Gamma_{\alpha}^{\sigma}\left\langle i\left|d_{\sigma}\right| i^{\prime}\right\rangle\left\langle j^{\prime}\left|d_{\sigma}^{\dagger}\right| j\right\rangle\left[f\left(E_{i^{\prime}}-E_{i}+\mu_{\alpha}^{\sigma}\right)\right] P_{i^{\prime} j^{\prime}} \\
& +\frac{1}{2} \sum_{i^{\prime} j^{\prime}} \sum_{\alpha \sigma} \Gamma_{\alpha}^{\sigma}\left\langle j^{\prime}\left|d_{\sigma}\right| j\right\rangle\left\langle i\left|d_{\sigma}^{\dagger}\right| i^{\prime}\right\rangle f\left(E_{i}-E_{i^{\prime}}-\mu_{\alpha}^{\sigma}\right) P_{i^{\prime} j^{\prime}} \\
& -\frac{1}{2} \sum_{i^{\prime} j^{\prime}} \sum_{\alpha \sigma} \Gamma_{\alpha}^{\sigma}\left\langle j^{\prime}\left|d_{\sigma}\right| i^{\prime}\right\rangle\left\langle i^{\prime}\left|d_{\sigma}^{\dagger}\right| j\right\rangle f\left(E_{i^{\prime}}-E_{j^{\prime}}-\mu_{\alpha}^{\sigma}\right) P_{i j^{\prime}} \\
& -\frac{1}{2} \sum_{i^{\prime} j^{\prime}} \sum_{\alpha \sigma} \Gamma_{\alpha}^{\sigma}\left\langle i^{\prime}\left|d_{\sigma}\right| j\right\rangle\left\langle j^{\prime}\left|d_{\sigma}^{\dagger}\right| i^{\prime}\right\rangle\left[f\left(E_{j^{\prime}}-E_{i^{\prime}}+\mu_{\alpha}^{\sigma}\right)\right] P_{i j^{\prime}} .
\end{aligned}
$$

The above equation is exactly Eq. (2) of Ref. 60 by replacing $i \rightarrow \alpha, j \rightarrow \beta, i^{\prime} \rightarrow \alpha^{\prime}$, and $j^{\prime} \rightarrow \beta^{\prime}$.

In this work, we have calculated three physical quantities: the total magnetization, the LUMO occupation, and the current through SMM. The general form of $\sigma$ current flowing from lead to SMM obtained by Lehmann and Loss can be rewritten using our notation as ${ }^{60}$

$$
\begin{aligned}
I_{\alpha}^{\sigma}= & e \operatorname{Re} \sum_{i i^{\prime} j} \Gamma_{\alpha}^{\sigma}\left\{f\left(E_{i}-E_{i^{\prime}}+\mu_{\alpha}^{\sigma}\right)\left\langle j\left|d_{\sigma}^{\dagger}\right| i^{\prime}\right\rangle\left\langle i^{\prime}\left|d_{\sigma}\right| i\right\rangle\right. \\
& \left.-f\left(E_{i^{\prime}}-E_{i}-\mu_{\alpha}^{\sigma}\right)\left\langle j\left|d_{\sigma}\right| i^{\prime}\right\rangle\left\langle i^{\prime}\left|d_{\sigma}^{\dagger}\right| i\right\rangle\right\} P_{i j} .
\end{aligned}
$$

As discussed in Sec. III B, we neglect two high-energy branches $|1, m\rangle^{+}$and $|2, m\rangle$ and consider only two lowenergy branches $|0, m\rangle$ and $|1, m\rangle^{-}$. In this context, the terms such as $\left\langle j\left|d_{\sigma}\right| i^{\prime}\right\rangle\left\langle i^{\prime}\left|d_{\sigma}^{\dagger}\right| i\right\rangle$ must require that $i=j$, e.g., if $\left|i^{\prime}\right\rangle=|1, m\rangle^{-}$, only $\left\langle 0, m-\sigma\left|d_{\sigma}\right| 1, m\right\rangle^{-}$and ${ }^{-}\left\langle 1, m\left|d_{\sigma}^{\dagger}\right| 0, m-\sigma\right\rangle$ are nonzero, so both $|i\rangle$ and $|j\rangle$ can only be $|0, m-\sigma\rangle$. As a result, Eq. (A15) reduces to 


$$
\begin{aligned}
I_{\alpha}^{\sigma}= & e \sum_{i i^{\prime}} \Gamma_{\alpha}^{\sigma}\left\{f\left(E_{i}-E_{i^{\prime}}+\mu_{\alpha}^{\sigma}\right)\left|\left\langle i^{\prime}\left|d_{\sigma}\right| i\right\rangle\right|^{2}-f\left(E_{i^{\prime}}-E_{i}-\mu_{\alpha}^{\sigma}\right)\right. \\
& \left.\times\left|\left\langle i\left|d_{\sigma}\right| i^{\prime}\right\rangle\right|^{2}\right\} P_{i},
\end{aligned}
$$

which can be further simplified as ${ }^{37}$

$$
I_{\alpha}^{\sigma}=-e \sum_{i j}\left(n_{j}-n_{i}\right) R_{i \rightarrow j}^{\alpha \sigma} P_{i}
$$

The LUMO occupation $N$ and the total magnetization $M$ involve only the diagonal terms of density matrix

$$
N=\operatorname{Tr}_{M}\left(\hat{\rho}_{M} \sum_{\sigma} n_{\sigma}\right)=\sum_{i j k} P_{i j}\left(\sum_{\sigma} n_{\sigma}\right)_{k i}=\sum_{i} P_{i}\left(\sum_{\sigma} n_{\sigma}\right)_{i i}
$$

and

$$
M=\operatorname{Tr}_{M}\left[\hat{\rho}_{M}\left(S_{z}+s_{z}\right)\right]=\sum_{i j k} P_{i j}\left(S_{z}+s_{z}\right)_{k i}=\sum_{i} P_{i}\left(S_{z}+s_{z}\right)_{i i}
$$

because $\left(s_{z}+S_{z}\right)$ and $\Sigma_{\sigma} n_{\sigma}$ are eigenoperators of $H_{\mathrm{SMM}}$.

Therefore, one only needs to know the diagonal terms of the density matrix. By letting $j=i$ in Eq. (A14) and employing the property $\left\langle i\left|d_{\sigma}\right| j^{\prime}\right\rangle\left\langle j^{\prime}\left|d_{\sigma}^{\dagger}\right| i^{\prime}\right\rangle \Rightarrow i=i^{\prime}$, one can readily show that the equation of $P_{i}$ only couples to other diagonal terms of density matrix.

Note that the property $\left\langle i\left|d_{\sigma}\right| j^{\prime}\right\rangle\left\langle j^{\prime}\left|d_{\sigma}^{\dagger}\right| i^{\prime}\right\rangle \Rightarrow i=i^{\prime}$ will be weakly violated in the presence of the transverse anisotropy $B_{2}$ and $B_{4}$, so that terms such as ${ }_{p}^{-}\left\langle 1, m\left|d_{\sigma}^{\dagger}\right| 0, m-\sigma^{\prime}\right\rangle_{p}$ may be also nonzero for $\left|\sigma^{\prime}\right|>|\sigma|$. However, the relative probability of these terms ${ }^{25} \sim\left(B_{2} / D\right)^{2},\left(B_{4} / D\right)^{2}$, thus can be omitted for $B_{2}, B_{4} \ll D$.

\section{APPENDIX B: PERTURBATION OF TRANSVERSE ANISOTROPY}

If we denote $H_{\mathrm{SMM}}=H^{(0)}+H^{\prime}$, the unperturbed eigenstates of $H^{(0)}$ by $\psi_{i}^{(0)}$, the first-order correction to $\psi_{i}^{(0)}$ is given by

$$
\psi_{i}^{(1)}=\sum_{j \neq i} \frac{H_{j i}^{\prime}}{E_{i}^{(0)}-E_{j}^{(0)}} \psi_{j}^{(0)},
$$

where $E_{i}^{(0)}$ is the unperturbed energy of $\psi_{i}^{(0)}$ and $H_{j i}^{\prime}=\left\langle\psi_{j}^{(0)}\left|H^{\prime}\right| \psi_{i}^{(0)}\right\rangle$. The second-order (the first order is zero for the present problem) correction to the energy is given by

$$
E_{i}^{(2)}=\sum_{j \neq i} \frac{\left|H_{j i}^{\prime}\right|^{2}}{E_{i}^{(0)}-E_{j}^{(0)}} .
$$

Finally, the perturbed states and their energies are obtained as $\psi=\psi^{(0)}+\psi^{(1)}$ and $E=E^{(0)}+E^{(2)}$. Because $|0, m\rangle$ and $|1, m\rangle^{-}$ are not coupled by $H^{\prime}$, we will discuss them separately.

\section{Branch $|0, m\rangle$}

The unperturbed energy is given by

$$
E_{|0, m\rangle}^{(0)}=-D m^{2},
$$

and the perturbation

$$
\begin{aligned}
\left\langle 0, m^{\prime}\left|H^{\prime}\right| 0, m\right\rangle= & -B_{2} \sqrt{(S-m)(S+m+1)(S-m-1)(S+m+2)} \delta_{m^{\prime}=m+2}-B_{2} \sqrt{(S+m)(S-m+1)(S+m-1)(S-m+2)} \delta_{m^{\prime}=m-2} \\
& -B_{4} \sqrt{(S-m)(S+m+1)(S-m-1)(S+m+2)} \sqrt{(S-m-2)(S+m+3)(S-m-3)(S+m+4)} \delta_{m^{\prime}=m+4} \\
& -B_{4} \sqrt{(S+m)(S-m+1)(S+m-1)(S-m+2)} \sqrt{(S+m-2)(S-m+3)(S+m-3)(S-m+4)} \delta_{m^{\prime}=m-4} . \quad(\mathrm{B} 4)
\end{aligned}
$$

However, because degenerate states $|0, \pm 1\rangle$ and $|0, \pm 2\rangle$ are coupled by $H^{\prime}$, Eqs. (B1) and (B2) cannot be applied directly. One has to perform a linear transformation first $\Psi_{|0, m\rangle}^{(0)}=P \psi_{|0, m\rangle}^{(0)}$ so that

$$
\begin{aligned}
& \left\langle\Psi_{|0,-1\rangle}^{(0)}\left|H^{(0)}\right| \Psi_{|0,-1\rangle}^{(0)}\right\rangle \neq\left\langle\Psi_{|0,1\rangle}^{(0)}\left|H^{(0)}\right| \Psi_{|0,1\rangle}^{(0)}\right\rangle, \\
& \left\langle\Psi_{|0,-2\rangle}^{(0)}\left|H^{(0)}\right| \Psi_{|0,-2\rangle}^{(0)}\right\rangle \neq\left\langle\Psi_{|0,2\rangle}^{(0)}\left|H^{(0)}\right| \Psi_{|0,2\rangle}^{(0)}\right\rangle
\end{aligned}
$$

and

$$
\begin{aligned}
& \left\langle\Psi_{|0,-1\rangle}^{(0)}\left|H^{\prime}\right| \Psi_{|0,1\rangle}^{(0)}\right\rangle=\left\langle\Psi_{|0,1\rangle}^{(0)}\left|H^{\prime}\right| \Psi_{|0,-1\rangle}^{(0)}\right\rangle=0, \\
& \left\langle\Psi_{|0,-2\rangle}^{(0)}\left|H^{\prime}\right| \Psi_{|0,2\rangle}^{(0)}\right\rangle=\left\langle\Psi_{|0,2\rangle}^{(0)}\left|H^{\prime}\right| \Psi_{|0,-2\rangle}^{(0)}\right\rangle=0 .
\end{aligned}
$$

Under the same linear transformation, $H^{(0)}$ and $H^{\prime}$ become

$$
\bar{H}^{(0)}=P^{-1} H^{(0)} P, \quad \bar{H}^{\prime}=P^{-1} H^{\prime} P .
$$

Then one replaces $H^{(0)} \rightarrow \bar{H}^{(0)}, H^{\prime} \rightarrow \bar{H}^{\prime}$, and $\psi_{i}^{(0)} \rightarrow \Psi_{i}^{(0)}$ in Eqs. (B1) and (B2) to perform the perturbation. 


\section{Branch $|1, m\rangle^{ \pm}$}

Because $|1, m\rangle^{-}$and $|1, m\rangle^{+}$together constitute the complete set of the subspace of $n=1$, the perturbation of $|1, m\rangle^{-}$has to take $|1, m\rangle^{+}$into account. The unperturbed states and their energy are obtained by diagonalizing

$$
\begin{aligned}
& \left(\begin{array}{c}
\mathrm{LUMO}_{\mathrm{LUMO}}\left\langle m+1 /\left.2\right|_{\mathrm{GS}}\left\langle\downarrow-1 /\left.2\right|_{\mathrm{GS}}\left\langle\uparrow\left|H^{(0)}\right| \downarrow\right\rangle\right\rangle_{\mathrm{LUMO}} \mid m+1 / 2\right\rangle_{\mathrm{GS}}\langle\rangle_{\mathrm{LUMO}}\langle m+1 / 2\rangle_{\mathrm{GS} \text { LUMO }}\left\langle m+1 /\left.2\right|_{\mathrm{GS}}\left\langle\downarrow\left|H^{(0)}\right| \uparrow\right\rangle_{\mathrm{LUMO}}|m-1 / 2|_{\mathrm{GS}}\left\langle\uparrow\left|H^{(0)}\right| \uparrow\right\rangle_{\mathrm{LUMO}} \mid m-1 / 2\right\rangle_{\mathrm{GS}} \\
\mathrm{GS}
\end{array}\right) \\
& =\left(\begin{array}{cc}
\epsilon_{0}-e V_{g}+\frac{J}{2} m+\frac{J}{4}-(D+\delta D)\left(m+\frac{1}{2}\right)^{2} & -\frac{1}{2} J \sqrt{S(S+1)-\left(m+\frac{1}{2}\right)\left(m-\frac{1}{2}\right)} \\
-\frac{1}{2} J \sqrt{S(S+1)-\left(m+\frac{1}{2}\right)\left(m-\frac{1}{2}\right)} & \epsilon_{0}-e V_{g}-\frac{J}{2} m+\frac{J}{4}-(D+\delta D)\left(m-\frac{1}{2}\right)^{2}
\end{array}\right)
\end{aligned}
$$

for $m \in\left\{-S+\frac{1}{2}, S-\frac{1}{2}\right\}$, so that in each subspace of $m$,

$$
|1, m\rangle^{ \pm}=\alpha_{m}^{ \pm}|\downarrow\rangle\left|m+\frac{1}{2}\right\rangle+\beta_{m}^{ \pm}|\uparrow\rangle\left|m-\frac{1}{2}\right\rangle
$$

and $\alpha_{|1, S+1 / 2\rangle}=\beta_{|1,-S-1 / 2\rangle}=0$ and $\alpha_{|1,-S-1 / 2\rangle}=\beta_{|1, S+1 / 2\rangle}=1$.

The perturbation is then given by

$$
\begin{aligned}
& \nu^{\prime}\left\langle 1, m^{\prime}\left|H^{\prime}\right| 1, m\right\rangle^{\nu}=\left[( \alpha _ { m ^ { \prime } } ^ { \nu ^ { \prime } } ) * \left\langlem^{\prime}+\frac{1}{2}\left|\left\langle\downarrow \left|+\left(\beta_{m^{\prime}}^{\nu^{\prime}}\right)^{*}\left\langle m^{\prime}-\frac{1}{2}|\langle\uparrow|] H^{\prime}\left[\alpha_{m}^{\nu}|\downarrow\rangle\left|m+\frac{1}{2}\right\rangle+\beta_{m}^{\nu}|\uparrow\rangle\left|m-\frac{1}{2}\right\rangle\right]\right.\right.\right.\right.\right.\right. \\
& =-\delta_{m^{\prime}=m+2} B_{2}\left[\left(\alpha_{m^{\prime}}^{\nu^{\prime}}\right)^{*} \alpha_{m}^{\nu} \sqrt{\left(S-m-\frac{1}{2}\right)\left(S+m+1 \frac{1}{2}\right)\left(S-m-1 \frac{1}{2}\right)\left(S+m+2 \frac{1}{2}\right)}\right. \\
& +\left(\beta_{m^{\prime}}^{\nu^{\prime}}\right)^{*} \beta_{m}^{\nu} \sqrt{\left.\left(S-m+\frac{1}{2}\right)\left(S+m+\frac{1}{2}\right)\left(S-m-\frac{1}{2}\right)\left(S+m+1 \frac{1}{2}\right)\right]} \\
& -\delta_{m^{\prime}=m-2} B_{2}\left[\left(\alpha_{m^{\prime}}^{\nu^{\prime}}\right)^{*} \alpha_{m}^{\nu} \sqrt{\left(S+m+\frac{1}{2}\right)\left(S-m+\frac{1}{2}\right)\left(S+m-\frac{1}{2}\right)\left(S-m+1 \frac{1}{2}\right)}\right. \\
& +\left(\beta_{m^{\prime}}^{\nu^{\prime}}\right)^{*} \beta_{m}^{\nu} \sqrt{\left.\left(S+m-\frac{1}{2}\right)\left(S-m+1 \frac{1}{2}\right)\left(S+m-1 \frac{1}{2}\right)\left(S-m+2 \frac{1}{2}\right)\right]} \\
& -\delta_{m^{\prime}=m+4} B_{4}\left[\left(\alpha_{m^{\prime}}^{\nu^{\prime}}\right)^{*} \alpha_{m}^{\nu} \sqrt{\left(S-m-\frac{1}{2}\right)\left(S+m+1 \frac{1}{2}\right)\left(S-m-1 \frac{1}{2}\right)\left(S+m+2 \frac{1}{2}\right)}\right. \\
& \times \sqrt{\left(S-m-2 \frac{1}{2}\right)\left(S+m+3 \frac{1}{2}\right)\left(S-m-3 \frac{1}{2}\right)\left(S+m+4 \frac{1}{2}\right)} \\
& +\left(\beta_{m^{\prime}}^{\nu^{\prime}}\right)^{*} \beta_{m}^{\nu} \sqrt{\left(S-m+\frac{1}{2}\right)\left(S+m+\frac{1}{2}\right)\left(S-m-\frac{1}{2}\right)\left(S+m+1 \frac{1}{2}\right)} \\
& \times \sqrt{\left.\left(S-m-1 \frac{1}{2}\right)\left(S+m+2 \frac{1}{2}\right)\left(S-m-2 \frac{1}{2}\right)\left(S+m+3 \frac{1}{2}\right)\right]} \\
& -\delta_{m^{\prime}=m-4} B_{4}\left[\left(\alpha_{m^{\prime}}^{\nu^{\prime}}\right)^{*} \alpha_{m}^{\nu} \sqrt{\left(S+m+\frac{1}{2}\right)\left(S-m+\frac{1}{2}\right)\left(S+m-\frac{1}{2}\right)\left(S-m+1 \frac{1}{2}\right)}\right. \\
& \times \sqrt{\left(S+m-1 \frac{1}{2}\right)\left(S-m+2 \frac{1}{2}\right)\left(S+m-2 \frac{1}{2}\right)\left(S-m+3 \frac{1}{2}\right)}+\left(\beta_{m^{\prime}}^{\nu^{\prime}}\right)^{*} \beta_{m}^{\nu} \\
& \times \sqrt{\left(S+m-\frac{1}{2}\right)\left(S-m+1 \frac{1}{2}\right)\left(S+m-1 \frac{1}{2}\right)\left(S-m+2 \frac{1}{2}\right)} \\
& \times \sqrt{\left.\left(S+m-2 \frac{1}{2}\right)\left(S-m+3 \frac{1}{2}\right)\left(S+m-3 \frac{1}{2}\right)\left(S-m+4 \frac{1}{2}\right)\right]} .
\end{aligned}
$$

Notice that here $m$ are half integers, so there is not degeneracy problem. One can employ Eqs. (B1) and (B2) directly. 
${ }^{1}$ L. Berger, Phys. Rev. B 54, 9353 (1996).

${ }^{2}$ J. C. Slonczewski, J. Magn. Magn. Mater. 159, L1 (1996).

${ }^{3}$ M. Tsoi, A. G. M. Jansen, J. Bass, W. C. Chiang, M. Seck, V. Tsoi, and P. Wyder, Phys. Rev. Lett. 80, 4281 (1998).

${ }^{4}$ E. B. Myers, D. C. Ralph, J. A. Katine, R. N. Louie, and R. A. Buhrman, Science 285, 867 (1999).

${ }^{5}$ J. A. Katine, F. J. Albert, R. A. Buhrman, E. B. Myers, and D. C. Ralph, Phys. Rev. Lett. 84, 3149 (2000).

${ }^{6}$ T. Kimura, Y. Otani, and J. Hamrle, Phys. Rev. Lett. 96, 037201 (2006).

${ }^{7}$ T. Yang, T. Kimura, and Y. Otani, Nat. Phys. 4, 851 (2008).

${ }^{8}$ M. Johnson and R. H. Silsbee, Phys. Rev. Lett. 55, 1790 (1985).

${ }^{9}$ F. J. Jedema, A. T. Filip, and B. J. van Wees, Nature (London) 410, 345 (2001).

${ }^{10}$ F. J. Jedema, H. B. Heersche, A. T. Filip, J. J. A. Baselmans, and B. J. van Wees, Nature (London) 416, 713 (2002).

${ }^{11}$ S. O. Valenzuela and M. Tinkham, Nature (London) 442, 176 (2006).

${ }^{12}$ R. Sessoli, D. Gatteschi, A. Caneschi, and M. A. Novak, Nature (London) 365, 141 (1993).

${ }^{13}$ D. Gatteschi, A. Caneschi, L. Pardi, and R. Sessoli, Science 265, 1054 (1994)

${ }^{14}$ J. R. Friedman, M. P. Sarachik, J. Tejada, and R. Ziolo, Phys. Rev. Lett. 76, 3830 (1996).

${ }^{15}$ L. Thomas, F. Lionti, R. Ballou, D. Gatteschi, R. Sessoli, and B. Barbara, Nature (London) 383, 145 (1996).

${ }^{16}$ A. L. Barra, D. Gatteschi, and R. Sessoli, Phys. Rev. B 56, 8192 (1997).

${ }^{17}$ C. Sangregorio, T. Ohm, C. Paulsen, R. Sessoli, and D. Gatteschi, Phys. Rev. Lett. 78, 4645 (1997).

${ }^{18}$ W. Wernsdorfer and R. Sessoli, Science 284, 133 (1999).

${ }^{19}$ D. Gatteschi, R. Sessoli, and J. Villian, Molecular Nanomagnets (Oxford University Press, New York, 2006).

${ }^{20}$ H. B. Heersche, Z. de Groot, J. A. Folk, H. S. J. van der Zant, C. Romeike, M. R. Wegewijs, L. Zobbi, D. Barreca, E. Tondello, and A. Cornia, Phys. Rev. Lett. 96, 206801 (2006).

${ }^{21}$ M.-H. Jo, J. E. Grose, K. Baheti, M. M. Deshmukh, J. J. Sokol, E. M. Rumberger, D. N. Hendrickson, J. R. Long, H. Park, and D. C. Ralph, Nano Lett. 6, 2014 (2006).

${ }^{22}$ C. Ni, S. Shah, D. Hendrickson, and P. R. Bandaru, Appl. Phys. Lett. 89, 212104 (2006).

${ }^{23}$ J. J. Henderson, C. M. Ramsey, E. del Barcoa, A. Mishra, and G. Christou, J. Appl. Phys. 101, 09E102 (2007).

${ }^{24}$ G.-H. Kim and T.-S. Kim, Phys. Rev. Lett. 92, 137203 (2004).

${ }^{25}$ C. Romeike, M. R. Wegewijs, and H. Schoeller, Phys. Rev. Lett. 96, 196805 (2006).

${ }^{26}$ C. Romeike, M. R. Wegewijs, M. Ruben, W. Wenzel, and H. Schoeller, Phys. Rev. B 75, 064404 (2007).

${ }^{27}$ C. Timm, Phys. Rev. B 76, 014421 (2007).

${ }^{28}$ C. Romeike, M. R. Wegewijs, W. Hofstetter, and H. Schoeller, Phys. Rev. Lett. 96, 196601 (2006).

${ }^{29}$ M. N. Leuenberger and E. R. Mucciolo, Phys. Rev. Lett. 97, 126601 (2006).

${ }^{30}$ C. Romeike, M. R. Wegewijs, W. Hofstetter, and H. Schoeller, Phys. Rev. Lett. 97, 206601 (2006).

${ }^{31}$ M. D. Nuñez Regueiro, P. S. Cornaglia, G. Usaj, and C. A. Balseiro, Phys. Rev. B 76, 075425 (2007).

${ }^{32}$ G. González, M. N. Leuenberger, and E. R. Mucciolo, Phys. Rev. B 78, 054445 (2008).
${ }^{33}$ G. González and M. N. Leuenberger, Phys. Rev. Lett. 98, 256804 (2007).

${ }^{34}$ K.-I. Imura, Y. Utsumi, and T. Martin, Phys. Rev. B 75, 205341 (2007).

${ }^{35}$ J. Lehmann, A. Gaita-Ario, E. Coronado, and D. Loss, Nat. Nanotechnol. 2, 312 (2007).

${ }^{36}$ F. Elste and C. Timm, Phys. Rev. B 75, 195341 (2007).

${ }^{37}$ C. Timm and F. Elste, Phys. Rev. B 73, 235304 (2006).

${ }^{38}$ F. Elste and C. Timm, Phys. Rev. B 73, 235305 (2006).

${ }^{39}$ M. Misiorny and J. Barnaś, Europhys. Lett. 78, 27003 (2007).

${ }^{40}$ M. Misiorny and J. Barnaś, Phys. Rev. B 75, 134425 (2007).

${ }^{41}$ M. Misiorny and J. Barnaś, Phys. Rev. B 76, 054448 (2007).

${ }^{42}$ M. Misiorny and J. Barnaś, Phys. Rev. B 77, 172414 (2008).

${ }^{43}$ M. N. Leuenberger and D. Loss, Nature (London) 410, 789 (2001).

${ }^{44}$ B. Zhou, R. Tao, S. Q. Shen, and J. Q. Liang, Phys. Rev. A 66, 010301(R) (2002).

${ }^{45}$ S. O. Valenzuela and M. Tinkham, J. Appl. Phys. 101, 09B103 (2007), and references therein.

${ }^{46}$ Y. Kato, R. C. Myers, A. C. Gossard, and D. D. Awschalom, Science 306, 1910 (2004).

${ }^{47}$ J. Wunderlich, B. Kaestner, J. Sinova, and T. Jungwirth, Phys. Rev. Lett. 94, 047204 (2005).

${ }^{48}$ X. Wang, G. E. W. Bauer, B. J. van Wees, A. Brataas, and Y. Tserkovnyak, Phys. Rev. Lett. 97, 216602 (2006).

${ }^{49}$ T. Moriyama, R. Cao, X. Fan, G. Xuan, B. K. Nikolić, Y. Tserkovnyak, J. Kolodzey, and J. Q. Xiao, Phys. Rev. Lett. 100, 067602 (2008).

${ }^{50}$ X. D. Cui, S. Q. Shen, J. Li, Y. Ji, W. K. Ge, and F. C. Zhang, Appl. Phys. Lett. 90, 242115 (2007).

${ }^{51}$ J. Li, X. Dai, S.-Q. Shen, and F. C. Zhang, Appl. Phys. Lett. 88, 162105 (2006).

${ }^{52}$ H. Zhao, X. Pan, A. L. Smirl, R. D. R. Bhat, A. Najmaie, J. E. Sipe, and H. M. van Driel, Phys. Rev. B 72, 201302(R) (2005).

${ }^{53}$ H. Zhao, E. J. Loren, H. M. van Driel, and A. L. Smirl, Phys. Rev. Lett. 96, 246601 (2006).

${ }^{54}$ H. Zhao, A. L. Smirl, and H. M. van Driel, Phys. Rev. B 75, 075305 (2007).

${ }^{55}$ H.-Z. Lu and S.-Q. Shen, Phys. Rev. B 77, 235309 (2008).

${ }^{56}$ D.-K. Wang, Q.-F. Sun, and H. Guo, Phys. Rev. B 69, 205312 (2004).

${ }^{57}$ Q.-F. Sun, Y. Xing, and S. Q. Shen, Phys. Rev. B 77, 195313 (2008).

${ }^{58}$ Y. Xing, Q.-F. Sun, and J. Wang, Appl. Phys. Lett. 93, 142107 (2008).

${ }^{59}$ G. Stefanucci, E. Perfetto, and M. Cini, Phys. Rev. B 78, 075425 (2008).

${ }^{60}$ J. Lehmann and D. Loss, Phys. Rev. Lett. 98, 117203 (2007).

${ }^{61}$ K. Park and M. R. Pederson, Phys. Rev. B 70, 054414 (2004).

${ }^{62}$ L. Michalak, C. M. Canali, M. R. Pederson, M. Paulsson, and V. G. Benza, arXiv:0812.1058 (unpublished).

${ }^{63}$ S. Wang, H.-L. Tsai, E. Libby, K. Folting, W. E. Streib, D. N. Hendrickson, and G. Christou, Inorg. Chem. 35, 7578 (1996).

${ }^{64}$ J.-M. Hu, Z.-D. Chen, and S.-Q. Shen, Phys. Rev. B 68, 104407 (2003).

${ }^{65}$ J. Koch and F. von Oppen, Phys. Rev. Lett. 94, 206804 (2005). 
${ }^{66}$ P. Politi, A. Rettori, F. Hartmann-Boutron, and J. Villain, Phys. Rev. Lett. 75, 537 (1995).

${ }^{67}$ K. Blum, Density Matrix Theory and Applications (Plenum, New York, 1996).
${ }^{68}$ A. N. Pasupathy, R. C. Bialczak, J. Martinek, J. E. Grose, L. A. K. Donev, P. L. McEuen, and D. C. Ralph, Science 306, 86 (2004).

${ }^{69}$ U. Fano, Rev. Mod. Phys. 29, 74 (1957). 